\title{
On Attractivity and Positivity of Solutions for Functional Integral Equations of Fractional Order
}

\author{
Xianyong Huang and Junfei Cao \\ Department of Mathematics, Guangdong University of Education, Guangzhou 510310, China \\ Correspondence should be addressed to Xianyong Huang; huangxianyong@gdei.edu.cn
}

Received 20 December 2012; Accepted 20 February 2013

Academic Editor: Sotiris Ntouyas

Copyright (C) 2013 X. Huang and J. Cao. This is an open access article distributed under the Creative Commons Attribution License, which permits unrestricted use, distribution, and reproduction in any medium, provided the original work is properly cited.

We investigate a class of functional integral equations of fractional order given by $x(t)=q(t)+f_{1}\left(t, x\left(\alpha_{1}(t)\right), x\left(\alpha_{2}(t)\right)\right)+$ $\left(f_{2}\left(t, x\left(\beta_{1}(t)\right), x\left(\beta_{2}(t)\right)\right) / \Gamma(\alpha)\right) \times \int_{0}^{t}(t-s)^{\alpha-1} f_{3}\left(t, s, x\left(\gamma_{1}(s)\right), x\left(\gamma_{2}(s)\right)\right) \mathrm{d} s$ : sufficient conditions for the existence, global attractivity, and ultimate positivity of solutions of the equations are derived. The main tools include the techniques of measures of noncompactness and a recent measure theoretic fixed point theorem of Dhage. Our investigations are placed in the Banach space of continuous and bounded real-valued functions defined on unbounded intervals. Moreover, two examples are given to illustrate our results.

\section{Introduction}

Nonlinear functional integral equations with bounded intervals have been studied extensively in the literature as regards various qualitative properties. This includes existence, uniqueness, stability, boundedness, monotonicity and extremality of solutions. But the study of nonlinear functional integral equations with unbounded intervals is relatively rare and exploited for the characteristics of attractivity and asymptotic attractivity of solutions. There are two methods for dealing with these characteristics of solutions, namely, classical fixed point theorems involving the hypotheses from analysis and topology and the fixed point theorems involving the use of measure of noncompactness. Each one of these methods has some advantages and disadvantages over the others [1-11].

The theory of integral equations of fractional order plays a very important role in describing some real world problems; it has recently received a lot of attention and now constitutes a significant branch of nonlinear analysis. In recent years, differential and integral equations of fractional order have found wide applications in physics, mechanics, engineering, electro chemistry, economics, and other fields [1219]; meanwhile, numerous research papers and monographs have appeared devoted to differential and integral equations of fractional order [20-38]. These papers contain various qualitative properties such as existence, uniqueness, stability, and asymptotic behavior for equations of fractional order.

The aim of this paper is to study the existence, global attractivity, and positivity of solutions for a functional integral equation of fractional order. The mentioned equation has rather general form and contains, as particular cases, a lot of fractional functional equations and nonlinear fractional integral equations of Volterra type. The main technique used in our considerations is the measures of noncompactness and a fixed point theorem of Dhage [3]. Our investigations will be situated in the Banach space of real functions which are defined, continuous, and bounded on the right-hand real half axis $\mathbb{R}_{+}$.

The measures of noncompactness used in this paper allow us not only to obtain the existence of solutions of the mentioned fractional functional integral equations but also to characterize those solutions in terms of global attractivity and positivity on unbounded intervals. This assertion means that all possible solutions of the equations in question are globally uniformly attractive and positive in the sense which will be defined further on.

It is worthwhile mentioning that the novelty of our approach consists mainly in the possibility of obtaining the global attractivity, asymptotic attractivity, and positivity of solutions for the considered fractional functional 
integral equations. We hope that the concept of measure of noncompactness considered here may be a stimulant for further investigations concerning solutions of nonlinear fractional differential and integral equations of other types.

\section{Notations, Definitions, and Auxiliary Facts}

This section is devoted to collect some notations, definitions, and auxiliary facts which will be used in the further considerations of this paper.

Let $E$ be a Banach space, $\mathscr{P}(E)$, a class of subsets of $E$ and let $\mathscr{P}_{p}(E)$ denote the class of all nonempty subsets of $E$ with property $p$. Here $p$ may be $p=\operatorname{closed}$ (in short $\mathrm{cl}$ ), $p=$ bounded (in short bd), $p=$ relatively compact (in short rcp), and so forth. Thus, $\mathscr{P}_{\mathrm{cl}}(E), \mathscr{P}_{\mathrm{bd}}(E), \mathscr{P}_{\mathrm{cl}, \mathrm{bd}}(E)$, and $\mathscr{P}_{\text {rcp }}(E)$ denote, respectively, the classes of closed, bounded, closed and bounded, and relatively compact subsets of $E$. A function $d_{H}: \mathscr{P}(E) \times \mathscr{P}(E) \rightarrow \mathbb{R}_{+}$defined by

$$
d_{H}(A, B)=\max \left\{\sup _{a \in A} D(a, B), \sup _{b \in B} D(b, A)\right\}
$$

satisfies all the conditions of a metric on $\mathscr{P}(E)$ and is called a Hausdorff-Pompeiu metric on $E$, where $D(a, B)=\inf \{\| a-$ $b \|: b \in B\}$. It is known that the hyperspace $\left(\mathscr{P}_{\mathrm{cl}}(E), d_{H}\right)$ is a complete metric space.

The auxiliary way of defining the measures of noncompactness has been adopted in several papers in the literature; see Akhmerov et al. [39], Appell [40], Banaś and Goebel [41], in the works Väth [42] and the references therein. In this paper, we adopt the following axiomatic definition of the measure of noncompactness in a Banach space given by Dhage [3]. The other useful forms appear in the work of Banaś and Goebel [41] and the references therein.

Before giving definition of measure of noncompactness, we need the following definitions.

Definition 1 (see [43]). A sequence $\left\{A_{n}\right\}$ of nonempty sets in $\mathscr{P}_{p}(E)$ is said to converge to a set $A$, called the limiting set if $d_{H}\left(A_{n}, A\right) \rightarrow 0$ as $n \rightarrow \infty$. A mapping $\mu: \mathscr{P}_{p}(E) \rightarrow \mathbb{R}_{+}$is called continuous if for any sequence $\left\{A_{n}\right\}$ in $\mathscr{P}_{p}(E)$ one has that

$$
d_{H}\left(A_{n}, A\right) \longrightarrow 0 \Longrightarrow\left|\mu\left(A_{n}\right)-\mu(A)\right| \longrightarrow 0 \text { as } n \longrightarrow \infty \text {. }
$$

Definition 2 (see [43]). A mapping $\mu: \mathscr{P}_{p}(E) \rightarrow \mathbb{R}_{+}$is called nondecreasing, if for $A, B \in \mathscr{P}_{p}(E)$ with $A \subseteq B$, then $\mu(A) \leq$ $\mu(B)$, where $\subseteq$ is an order relation by inclusion $\mathscr{P}_{p}(E)$.

Now we are equipped with the necessary details to define the measures of noncompactness for a bounded subset of the Banach space $E$.

Definition 3 (see [43]). A function $\mu: \mathscr{P}_{p}(E) \rightarrow \mathbb{R}_{+}$is called a measure of noncompactness if it satisfies $1^{o} \emptyset \neq \mu^{-1}(0) \subset$ $\mathscr{P}_{\text {rcp }}(E), 2^{o} \mu(A)=\mu(\bar{A})$, where $\bar{A}$ is the closure of $A$, $3^{o} \mu(A)=\mu(\operatorname{Conv}(A))$, where $\operatorname{Conv}(A)$ is the convex hull of $A$ and $4^{\circ} \mu$ is nondecreasing, and $5^{\circ}$ if $\left\{A_{n}\right\}$ is a decreasing sequence of sets in $\mathscr{P}_{\text {bd }}(E)$ such that $\lim _{n \rightarrow \infty}\left(A_{n}\right)=0$, then the limiting set $A_{\infty}=\lim _{n \rightarrow \infty} \bar{A}_{n}=\cap_{n=0}^{\infty} \bar{A}_{n}$ is nonempty.

The family ker $\mu$ described in $1^{o}$ is said to be the kernel of $\mu$ and

$$
\operatorname{ker} \mu=\left\{A \in \mathscr{P}_{\text {bd }}(E) \mid \mu(A)=0\right\} \subset \mathscr{P}_{\text {rcp }}(E) .
$$

A measure $\mu$ is called complete or full if the kernel ker $\mu$ of $\mu$ consists of all possible relatively compact subsets of $E$. Next, a measure $\mu$ is called sublinear if it satisfies

$$
\begin{gathered}
6^{o} \mu(\lambda A)=|\lambda| \mu(A) \quad \text { for } \lambda \in \mathbb{R}, \\
7^{o} \mu(A+B) \leq \mu(A)+\mu(B) .
\end{gathered}
$$

There do exist the sublinear measures of noncompactness on Banach spaces E. Indeed, the Kuratowskii and Hausdorff measures of noncompactness are sublinear in $E$. A good collection of different types of measures of noncompactness appears in Appell [40].

Observe that the limiting set $A_{\infty}$ from $5^{\circ}$ is a member of the family $\operatorname{ker} \mu$. In fact, since

$$
\mu\left(A_{\infty}\right) \leq \mu\left(\bar{A}_{n}\right)=\mu\left(A_{n}\right), \quad \text { for any } n,
$$

one infers that $\mu\left(A_{\infty}\right)=0$. This yields that $A_{\infty} \in \operatorname{ker} \mu$. This simple observation will be essential in our further investigations.

Now we state a key fixed point theorem of Dhage [3] which will be used in the sequel. Before stating this fixed point result, we give a useful definition.

Definition 4 (see [43]). A mapping $Q: E \rightarrow E$ is called $D$-set-Lipschitz if there exists a continuous nondecreasing function $\varphi: \mathbb{R}_{+} \rightarrow \mathbb{R}_{+}$such that $\mu(Q(A)) \leq \varphi(\mu(A))$ for all $A \in \mathscr{P}_{\text {bd }}(E)$ with $Q(A) \in \mathscr{P}_{\text {bd }}(E)$, where $\varphi(0)=0$. Sometimes we call the function $\varphi$ to be a $D$-function of $Q$ on $E$. In the special case, when $\varphi(r)=k r, k>0, Q$ is called a $k$-set-Lipschitz mapping, and if $k<1$, then $Q$ is called a $k$ set-contraction on $E$. Further, if $\varphi(r)<r$ for $r>0$, then $Q$ is called a nonlinear $D$-set-contraction on $E$.

Theorem 5 (see, Dhage [43]). Let $C$ be a nonempty, closed, convex, and bounded subset of a Banach space $E$ and let $Q$ : $C \rightarrow C$ be a continuous and nonlinear D-set-contraction. Then $Q$ has a fixed point.

Remark 6. Denote by $\operatorname{Fix}(Q)$ the set of all fixed points of the operator $Q$ which belong to $C$. It can be shown that the set $\operatorname{Fix}(Q)$ existing in Theorem 5 belongs to the family ker $\mu$. In fact if $\operatorname{Fix}(Q) \notin \operatorname{ker} \mu$, then $\mu(\operatorname{Fix}(Q))>0$ and $Q(\operatorname{Fix}(Q))=$ $\operatorname{Fix}(Q)$. Now from nonlinear $D$-set-contraction it follows that $\mu(Q(\operatorname{Fix}(Q))) \leq \varphi(\mu(\operatorname{Fix}(Q)))$ which is a contradiction, since $\varphi(r)<r$ for $r>0$. Hence, $\operatorname{Fix}(Q) \in \operatorname{ker} \mu$.

Our further considerations will be placed in the Banach space $B C\left(\mathbb{R}_{+}, \mathbb{R}\right)$ consisting of all real functions $x=$ $x(t)$ defined, continuous, and bounded on $\mathbb{R}_{+}$. This space is equipped with the standard supremum norm $\|x\|=$ $\sup \left\{|x(t)|: t \in \mathbb{R}_{+}\right\}$.

For our purposes we will use the Hausdorff or ball measure of noncompactness in $B C\left(\mathbb{R}_{+}, \mathbb{R}\right)$. A handy formula for 
Hausdorff measure of noncompactness useful in application is defined as follows. Fix a nonempty and bounded subset $X$ of the space $B C\left(\mathbb{R}_{+}, \mathbb{R}\right)$ and a positive number $T$. For $x \in X$ and $\varepsilon>0$, denote by $\omega^{T}(x, \varepsilon)$ the modulus of continuity of the function $x$ on the closed and bounded interval $[0, T]$ defined by

$$
\omega^{T}(x, \varepsilon)=\sup \{|x(t)-x(s)|: t, s \in[0, T],|t-s|<\varepsilon\} .
$$

Next, put

$$
\begin{aligned}
& \omega^{T}(X, \varepsilon)=\sup \left\{\omega^{T}(x, \varepsilon): x \in X\right\}, \\
& \omega_{0}^{T}(X)=\lim _{\varepsilon \rightarrow 0} \omega^{T}(X, \varepsilon) .
\end{aligned}
$$

It is known that $\omega_{0}^{T}$ is a measure of noncompactness in the Banach space $C([0, T], \mathbb{R})$ of continuous and real-valued functions defined on a closed and bounded interval $[0, T]$ in $\mathbb{R}$ which is equivalent to Hausdorff or ball measure $\chi$ of noncompactness in it. In fact, one has $\chi(X)=(1 / 2) \omega_{0}^{T}(X)$ for any bounded subset $X$ of $C([0, T], \mathbb{R})$ (see Banaś and Goebel [41] and the references therein). Finally, define $\omega_{0}(X)=$ $\lim _{T \rightarrow \infty} \omega_{0}^{T}(X)$.

Now, for a fixed number $t \in \mathbb{R}_{+}$, denote

$$
\begin{gathered}
X(t)=\{x(t): x \in X\}, \\
\|X(t)\|=\sup \{|x(t)|: x \in X\}, \\
\|X(t)-c\|=\sup \{|x(t)-c|: x \in X\} .
\end{gathered}
$$

Finally, consider the functions $\mu$ 's defined on the family $\mathscr{P}_{\text {cl,bd }}(X)$ by the formulas

$$
\begin{gathered}
\mu_{a}(X)=\max \left\{\omega_{0}(X), \limsup _{t \rightarrow \infty} \operatorname{diam} X(t)\right\}, \\
\mu_{b}(X)=\max \left\{\omega_{0}(X), \limsup _{t \rightarrow \infty}\|X(t)\|\right\}, \\
\mu_{c}(X)=\max \left\{\omega_{0}(X), \limsup _{t \rightarrow \infty}\|X(t)-c\|\right\} .
\end{gathered}
$$

Let $T>0$ be fixed. Then for any $x \in B C\left(\mathbb{R}_{+}, \mathbb{R}\right)$, define $\delta_{T}(x)=\sup \{|| x(t)|-x(t)|: t \geq T\}$. Similarly, for any bounded subset $X$ of $B C\left(\mathbb{R}_{+}, \mathbb{R}\right)$, define

$$
\begin{aligned}
& \delta_{T}(X)=\sup \left\{\delta_{T}(x): x \in X\right\}, \\
& \delta(X)=\lim _{T \rightarrow \infty} \delta_{T}(X) .
\end{aligned}
$$

Define the functions $\mu_{\mathrm{ad}}, \mu_{\mathrm{bd}}, \mu_{\mathrm{cd}}: \mathscr{P}_{\mathrm{bd}}(E) \rightarrow \mathbb{R}_{+}$by the formulas

$$
\begin{aligned}
& \mu_{\mathrm{ad}}(X)=\max \left\{\mu_{a}(X), \delta(X)\right\}, \\
& \mu_{\mathrm{bd}}(X)=\max \left\{\mu_{b}(X), \delta(X)\right\}, \\
& \mu_{\mathrm{cd}}(X)=\max \left\{\mu_{c}(X), \delta(X)\right\},
\end{aligned}
$$

for all $X \in \mathscr{P}_{\mathrm{cl}, \mathrm{bd}}(E)$.
Remark 7. It can be shown as in Banaś and Goebel [41] that the functions $\mu_{a}, \mu_{b}, \mu_{c}, \mu_{\mathrm{ad}}, \mu_{\mathrm{bd}}$, and $\mu_{\mathrm{cd}}$ are measures of noncompactness in the space $B C\left(\mathbb{R}_{+}, \mathbb{R}\right)$. The kernels $\operatorname{ker} \mu_{a}, \operatorname{ker} \mu_{b}$, and $\operatorname{ker} \mu_{c}$ of the measures $\mu_{a}, \mu_{b}$, and $\mu_{c}$ consist of nonempty and bounded subsets $X$ of $B C\left(\mathbb{R}_{+}, \mathbb{R}\right)$ such that functions from $X$ are locally equicontinuous on $\mathbb{R}_{+}$and the thickness of the bundle formed by functions from $X$ tends to zero at infinity. Moreover, the functions from ker $\mu_{c}$ come closer along a line $y(t)=c$ and the functions from ker $\mu_{b}$ come closer along the line $y(t)=$ 0 as $t$ increases to $\infty$ through $\mathbb{R}_{+}$. A similar situation is also true for the kernels $\operatorname{ker} \mu_{\mathrm{ad}}$, $\operatorname{ker} \mu_{\mathrm{bd}}$, and $\operatorname{ker} \mu_{\mathrm{cd}}$ of the measures of noncompactness $\mu_{\mathrm{ad}}, \mu_{\mathrm{bd}}$, and $\mu_{\mathrm{cd}}$. Moreover, these measures $\mu_{\mathrm{ad}}, \mu_{\mathrm{bd}}$, and $\mu_{\mathrm{cd}}$ characterize the ultimate positivity of the functions belonging to the kernels of ker $\mu_{\mathrm{ad}}$, $\operatorname{ker} \mu_{\mathrm{bd}}$, and $\operatorname{ker} \mu_{\mathrm{cd}}$. The above expressed property of ker $\mu_{a}$, $\operatorname{ker} \mu_{b}, \operatorname{ker} \mu_{c}$, and $\operatorname{ker} \mu_{\mathrm{ad}}, \operatorname{ker} \mu_{\mathrm{bd}}, \operatorname{ker} \mu_{\mathrm{cd}}$ permits us to characterize solutions of the fractional functional integral equations considered in the sequel.

In order to introduce further concepts used in this paper, let us assume that $E=B C\left(\mathbb{R}_{+}, \mathbb{R}\right)$ and let $\Omega$ be a subset of $X$. Let $Q: E \rightarrow E$ be an operator and consider the following operator equation in $E$ :

$$
Q x(t)=x(t), \quad \forall t \in \mathbb{R}_{+} .
$$

Below we give different characterizations of the solutions for (14) on $\mathbb{R}_{+}$.

Definition 8. We say that solutions of (14) are locally attractive if there exists a closed ball $B_{r}\left(x_{0}\right)$ in the space $B C\left(\mathbb{R}_{+}, \mathbb{R}\right)$ for some $x_{0} \in B C\left(\mathbb{R}_{+}, \mathbb{R}\right)$ such that for arbitrary solutions $x=x(t)$ and $y=y(t)$ of (14) belonging to $B_{r}\left(x_{0}\right) \cap \Omega$ one has that

$$
\lim _{t \rightarrow \infty}(x(t)-y(t))=0 .
$$

In the case when the limit (15) is uniform with respect to the set $B_{r}\left(x_{0}\right) \cap \Omega$, that is, when for each $\varepsilon>0$ there exists $T>0$ such that

$$
|x(t)-y(t)|<\varepsilon,
$$

for all $x, y \in B_{r}\left(x_{0}\right) \cap \Omega$ being solutions of (14) and for all $t \geq T$, we will say that solutions of (14) are uniformly locally attractive on $\mathbb{R}_{+}$.

Definition 9. The solution $x=x(t)$ of (14) is said to be globally attractive if (15) holds for each solution $y=y(t)$ of (14) on $\Omega$. In other words, we may say that solutions of (14) are globally attractive, if for arbitrary solutions $x(t)$ and $y(t)$ of (14) on $\Omega$, the condition (15) is satisfied. In the case when the condition (15) is satisfied uniformly with respect to the set $\Omega$, that is, if for every $\varepsilon>0$ there exists $T>0$ such that the inequality (16) is satisfied for all $x, y \in \Omega$ being the solutions of (14) and for all $t \geq T$, we will say that solutions of (14) are uniformly globally attractive on $\mathbb{R}_{+}$. 
The following definitions appear in the work of Dhage [7].

Definition 10. A line $y(t)=c$, where $c$ is a real number, is called an attractor for a solution $x \in B C\left(\mathbb{R}_{+}, \mathbb{R}\right)$ to (14) if $\lim _{t \rightarrow \infty}[x(t)-c]=0$. In this case the solution $x$ to (14) is also called to be asymptotic to the line $y(t)=c$ and the line is an asymptote for the solution $x$ on $\mathbb{R}_{+}$.

Now we introduce the following definitions which are useful in the sequel.

Definition 11. The solutions of (14) are said to be globally asymptotically attractive if for any two solutions $x=x(t)$ and $y=y(t)$ of (14), the condition (15) is satisfied, and there is a line which is a common attractor to them on $\mathbb{R}_{+}$. In the case when condition (15) is satisfied uniformly, that is, if for every $\varepsilon>0$ there exists $T>0$ such that the inequality (16) is satisfied for all $t \geq T$ and for all $x, y$ being the solutions of (14) and having a line as a common attractor, we will say that solutions of (14) are uniformly globally asymptotically attractive on $\mathbb{R}_{+}$.

Definition 12. A solution $x$ of (14) is called locally ultimately positive if there exists a closed ball $B_{r}\left(x_{0}\right)$ in $B C\left(\mathbb{R}_{+}, \mathbb{R}\right)$ for some $x_{0} \in B C\left(\mathbb{R}_{+}, \mathbb{R}\right)$ such that $x \in B_{r}\left(x_{0}\right)$ and

$$
\lim _{t \rightarrow \infty}|| x(t)|-x(t)|=0 .
$$

In the case when the limit (17) is uniform with respect to the solution set of the operator equation (14), that is, when for each $\varepsilon>0$ there exists $T>0$ such that

$$
|| x(t)|-x(t)|<\varepsilon
$$

for all $x$ being solutions of (14) and for all $t \geq T$, we will say that solutions of (14) are uniformly locally ultimately positive on $\mathbb{R}_{+}$.

Definition 13. A solution $x \in C\left(\mathbb{R}_{+}, \mathbb{R}\right)$ of (14) is called globally ultimately positive if (17) is satisfied. In the case when the limit (17) is uniform with respect to the solution set of the operator equation (14) in $C\left(\mathbb{R}_{+}, \mathbb{R}\right)$, that is, when for each $\varepsilon>0$ there exists $T>0$ such that (18) is satisfied for all $x$ being solutions of (14) and for all $t \geq T$, we will say that solutions of (14) are uniformly globally ultimately positive on $\mathbb{R}_{+}$.

Remark 14. Note that the global attractivity and global asymptotic attractivity imply, respectively, the local attractivity and local asymptotic attractivity of the solutions for the operator equation (14) on $\mathbb{R}_{+}$. Similarly, global ultimate positivity implies local ultimate positivity of the solutions for the operator equation (14) on unbounded intervals. However, the converse of the above two statements may not be true. A few details of ultimate positivity are given in the work of Dhage [44].

Finally, we introduce the concept of the fraction integral and the Riemann-Liouville fractional derivative.
Definition 15 (see $[45,46])$. The fractional integral of order $\alpha>0$ with the lower limit $t_{0}$ for a function $f$ is defined as

$$
I^{\alpha} f(t)=\frac{1}{\Gamma(\alpha)} \int_{t_{0}}^{t}(t-s)^{\alpha-1} f(s) \mathrm{d} s, \quad t>t_{0},
$$

provided the right-hand side is pointwise on $\left[t_{0}, \infty\right)$, where $\Gamma(\alpha)$ is the Gamma function.

Definition 16 (see $[45,46])$. The Riemann-Liouville derivative of order $\alpha>0$ with the lower limit $t_{0}$ for a function $f:\left[t_{0}, \infty\right) \rightarrow \mathbb{R}$ can be written as

$$
\begin{array}{r}
D_{t}^{\alpha} f(t)=\frac{1}{\Gamma(n-\alpha)} \frac{\mathrm{d}^{n}}{\mathrm{~d} t^{n}} \int_{t_{0}}^{t}(t-s)^{\alpha+1-n} f(s) \mathrm{d} s, \\
t>t_{0}, n-1<\alpha<n .
\end{array}
$$

The first and maybe the most important property of the Riemann-Liouville fractional derivative is that for $t>t_{0}$ and $\alpha>0$, one has $D^{\alpha}\left(I^{\alpha} f(t)\right)=f(t)$, which means that the Riemann-Liouville fractional differentiation operator is a left inverse to the Riemann-Liouville fractional integration operator of the same order $\alpha$. paper.

In the following section we prove the main results of this

\section{Attractivity and Positivity Results}

In this section we will investigate the following functional integral equation of fractional order with deviating arguments:

$$
\begin{aligned}
x(t)= & q(t)+f_{1}\left(t, x\left(\alpha_{1}(t)\right), x\left(\alpha_{2}(t)\right)\right) \\
& +\frac{f_{2}\left(t, x\left(\beta_{1}(t)\right), x\left(\beta_{2}(t)\right)\right)}{\Gamma(\alpha)} \\
& \times \int_{0}^{t}(t-s)^{\alpha-1} f_{3}\left(t, s, x\left(\gamma_{1}(s)\right), x\left(\gamma_{2}(s)\right)\right) \mathrm{d} s,
\end{aligned}
$$

where $t \in \mathbb{R}_{+}, q: \mathbb{R}_{+} \rightarrow \mathbb{R}, f_{1}, f_{2}: \mathbb{R}_{+} \times \mathbb{R} \times \mathbb{R} \rightarrow \mathbb{R}$, $f_{3}: \mathbb{R}_{+} \times \mathbb{R}_{+} \times \mathbb{R} \times \mathbb{R} \rightarrow \mathbb{R}, \alpha_{1}, \alpha_{2}, \beta_{1}, \beta_{2}, \gamma_{1}, \gamma_{2}: \mathbb{R}_{+} \rightarrow \mathbb{R}_{+}$, $\alpha \in(0,1)$, and $\Gamma(\alpha)$ denotes the Gamma function.

Equation (21) has rather general form, when

$$
\begin{gathered}
f_{1}\left(t, x\left(\alpha_{1}(t)\right), x\left(\alpha_{2}(t)\right)\right)=0, \\
f_{2}\left(t, x\left(\beta_{1}(t)\right), x\left(\beta_{2}(t)\right)\right)=f(t, x(t)), \\
f_{3}\left(t, s, x\left(\gamma_{1}(s)\right), x\left(\gamma_{2}(s)\right)\right)=u(t, s, x(t)) .
\end{gathered}
$$

Equation (21) reduces to the following quadratic Volterra integral equation of fractional order

$$
x(t)=q(t)+\frac{f(t, x(t))}{\Gamma(\alpha)} \int_{0}^{t}(t-s)^{\alpha-1} u(t, s, x(s)) \mathrm{d} s .
$$


Equation (23) has been studied in the work of Banaś and O'Regan [47] for the existence and local attractivity of solutions via classical hybrid fixed point theory, when

$$
\begin{gathered}
q(t)=0, \\
f_{1}\left(t, x\left(\alpha_{1}(t)\right), x\left(\alpha_{2}(t)\right)\right)=g(t, x(\eta(t))), \\
f_{2}\left(t, x\left(\beta_{1}(t)\right), x\left(\beta_{2}(t)\right)\right)=f(t, x(\beta(t))), \\
f_{3}\left(t, s, x\left(\gamma_{1}(s)\right), x\left(\gamma_{2}(s)\right)\right)=h(t, s) u(s, x(\gamma(s))) .
\end{gathered}
$$

Equation (21) reduces to the following functional integral equation of fractional order considered in Balachandran et al. [48] for the local attractivity of solutions

$$
\begin{aligned}
x(t)= & g(t, x(\eta(t)))+\frac{f(t, x(\beta(t)))}{\Gamma(\alpha)} \\
& \times \int_{0}^{t}(t-s)^{\alpha-1} h(t, s) u(s, x(\gamma(s))) \mathrm{d} s .
\end{aligned}
$$

Therefore, (21) is more general and contains as particular cases a lot of fractional functional equations and nonlinear fractional integral equations of Volterra type.

By a solution of $(21)$ we mean a function $x \in C\left(\mathbb{R}_{+}, \mathbb{R}\right)$ that satisfies (21), where $C\left(\mathbb{R}_{+}, \mathbb{R}\right)$ is the space of continuous real-valued functions defined on $\mathbb{R}_{+}$.

Equation (21) will be considered under the following assumptions.

$\left(H_{0}\right)$ the functions $\alpha_{1}, \alpha_{2}, \beta_{1}, \beta_{2}: \mathbb{R}_{+} \rightarrow \mathbb{R}_{+}$are continuous and satisfy

$$
\begin{aligned}
& \alpha_{1}(t), \alpha_{2}(t), \beta_{1}(t), \beta_{2}(t) \geq t, \\
& \alpha_{1}(t), \alpha_{2}(t), \beta_{1}(t), \beta_{2}(t) \longrightarrow \infty \text { as } t \longrightarrow \infty .
\end{aligned}
$$

$\left(H_{1}\right)$ the function $q: \mathbb{R}_{+} \rightarrow \mathbb{R}$ is continuous and bounded.

$\left(H_{2}\right)$ the function $f_{1}: \mathbb{R}_{+} \times \mathbb{R} \times \mathbb{R} \rightarrow \mathbb{R}$ is continuous and there exists a bounded function $\ell: \mathbb{R}_{+} \rightarrow \mathbb{R}$ with bound $L$ and a positive constant $M$ such that

$$
\begin{aligned}
& \left|f_{1}\left(t, x_{1}, y_{1}\right)-f_{1}\left(t, x_{2}, y_{2}\right)\right| \\
& \quad \leq \frac{\ell(t) \max \left\{\left|x_{1}-x_{2}\right|,\left|y_{1}-y_{2}\right|\right\}}{M+\max \left\{\left|x_{1}-x_{2}\right|,\left|y_{1}-y_{2}\right|\right\}},
\end{aligned}
$$

for all $t \in \mathbb{R}_{+}$and $x_{1}, x_{2}, y_{1}, y_{2} \in \mathbb{R}$. Moreover, we assume that $L \leq M$.

$\left(H_{3}\right)$ the function $f_{2}: \mathbb{R}_{+} \times \mathbb{R} \times \mathbb{R} \rightarrow \mathbb{R}$ is continuous and there exists a function $m: \mathbb{R}_{+} \rightarrow \mathbb{R}_{+}$being continuous on $\mathbb{R}_{+}$and such that

$$
\begin{aligned}
& \left|f_{2}\left(t, x_{1}, y_{1}\right)-f_{2}\left(t, x_{2}, y_{2}\right)\right| \\
& \quad \leq m(t)\left(\left|x_{1}-x_{2}\right|+\left|y_{1}-y_{2}\right|\right),
\end{aligned}
$$

for all $t \in \mathbb{R}_{+}$and $x_{1}, x_{2}, y_{1}, y_{2} \in \mathbb{R}$.

$\left(H_{4}\right)$ the function $F_{1}(t)=f_{1}(t, 0,0)$ is bounded with $F_{1}=$ $\sup \left\{\left|f_{1}(t, 0,0)\right|: t \in \mathbb{R}_{+}\right\}$.
$\left(H_{5}\right)$ the function $f_{3}: \mathbb{R}_{+} \times \mathbb{R}_{+} \times \mathbb{R} \times \mathbb{R} \rightarrow \mathbb{R}$ is continuous and there exists a function $n: \mathbb{R}_{+} \rightarrow \mathbb{R}_{+}$being continuous on $\mathbb{R}_{+}$and a function $\varphi: \mathbb{R}_{+} \rightarrow \mathbb{R}_{+}$being continuous and nondecreasing on $\mathbb{R}_{+}$with $\varphi(0)=0$ such that

$$
\begin{aligned}
& \left|f_{3}\left(t, s, x_{1}, y_{1}\right)-f_{3}\left(t, s, x_{2}, y_{2}\right)\right| \\
& \quad \leq n(t) \varphi\left(\left|x_{1}-x_{2}\right|+\left|y_{1}-y_{2}\right|\right),
\end{aligned}
$$

for all $t \in \mathbb{R}_{+}$and $x_{1}, x_{2}, y_{1}, y_{2} \in \mathbb{R}$.

For further purposes, define the functions $F_{2}: \mathbb{R}_{+} \rightarrow \mathbb{R}_{+}$ by putting $F_{2}(t)=f_{2}(t, 0,0)$ and $F_{3}: \mathbb{R}_{+} \rightarrow \mathbb{R}_{+}$by putting $F_{3}(t)=\max \left\{\left|f_{3}(t, s, 0,0)\right|: 0 \leq s \leq t\right\}$. Obviously the functions $F_{2}$ and $F_{3}$ are continuous on $\mathbb{R}_{+}$.

$\left(H_{6}\right)$ the functions $a, b, c, d: \mathbb{R}_{+} \rightarrow \mathbb{R}_{+}$defined by the formulas

$$
\begin{array}{ll}
a(t)=2 m(t) n(t) t^{\alpha}, & b(t)=2 m(t) F_{3}(t) t^{\alpha}, \\
c(t)=n(t)\left|F_{2}(t)\right| t^{\alpha}, & d(t)=\left|F_{2}(t)\right| F_{3}(t) t^{\alpha}
\end{array}
$$

are bounded on $\mathbb{R}_{+}$and the functions $a(t), b(t), c(t), d(t)$ vanish at infinity, that is,

$$
\lim _{t \rightarrow \infty} a(t)=\lim _{t \rightarrow \infty} b(t)=\lim _{t \rightarrow \infty} c(t)=\lim _{t \rightarrow \infty} d(t)=0 .
$$

Keeping in mind assumption $\left(H_{6}\right)$, define the following finite constants:

$$
\begin{array}{ll}
A=\sup \left\{a(t): t \in \mathbb{R}_{+}\right\}, & B=\sup \left\{b(t): t \in \mathbb{R}_{+}\right\}, \\
C=\sup \left\{c(t): t \in \mathbb{R}_{+}\right\}, & D=\sup \left\{d(t): t \in \mathbb{R}_{+}\right\} .
\end{array}
$$

Now we formulate the last assumption.

$\left(H_{7}\right)$ There exists a positive solution $r_{0}$ of the inequality

$$
\|q\|+\frac{L r}{M+r}+F_{1}+\frac{\operatorname{Ar\varphi }(2 r)+B r+C \varphi(2 r)+D}{\Gamma(\alpha+1)}<r .
$$

Remark 17. Hypothesis $\left(\mathrm{H}_{2}\right)$ is satisfied if in particular $f_{1}$ satisfies the condition,

$$
\begin{aligned}
& \left|f_{1}\left(t, x_{1}, y_{1}\right)-f_{1}\left(t, x_{2}, y_{2}\right)\right| \\
& \quad \leq \frac{\ell(t)\left[\left|x_{1}-x_{2}\right|+\left|y_{1}-y_{2}\right|\right]}{2 M+\left[\left|x_{1}-x_{2}\right|+\left|y_{1}-y_{2}\right|\right]}
\end{aligned}
$$

for all $t \in \mathbb{R}_{+}$and $x_{1}, x_{2}, y_{1}, y_{2} \in \mathbb{R}$, where $L \leq M$, and the function $\ell$ is defined as in hypothesis $\left(H_{2}\right)$ which further yields the usual Lipschitz condition on the function $f_{1}$,

$$
\left|f_{1}\left(t, x_{1}, y_{1}\right)-f_{1}\left(t, x_{2}, y_{2}\right)\right| \leq \frac{\ell(t)}{2 M}\left[\left|x_{1}-x_{2}\right|+\left|y_{1}-y_{2}\right|\right],
$$

for all $t \in \mathbb{R}_{+}$and $x_{1}, x_{2}, y_{1}, y_{2} \in \mathbb{R}$ provided $L<M$. As mentioned in the work of Dhage [11], our hypothesis $\left(H_{2}\right)$ is more general than that existing in the literature. 
Now, consider the operators $F, G, U$, and $Q$ defined on the space $B C\left(\mathbb{R}_{+}, \mathbb{R}\right)$ :

$$
\begin{gathered}
(G x)(t)=q(t)+f_{1}\left(t, x\left(\alpha_{1}(t)\right),\left(\alpha_{2}(t)\right)\right), \\
(F x)(t)=f_{2}\left(t, x\left(\beta_{1}(t)\right),\left(\beta_{2}(t)\right)\right), \\
(U x)(t)=\frac{1}{\Gamma(\alpha)} \int_{0}^{t}(t-s)^{\alpha-1} f_{3}\left(t, s, x\left(\gamma_{1}(s)\right), x\left(\gamma_{2}(s)\right)\right) \mathrm{d} s, \\
(Q x)(t)=(G x)(t)+(F x)(t)(U x)(t) .
\end{gathered}
$$

Then one has the following lemma.

Lemma 18. Under the above assumptions the operator $Q$ transforms the ball $B_{r_{0}}$ in the space $B C\left(\mathbb{R}_{+}, \mathbb{R}\right)$ into itself. Moreover, all solutions of $(21)$ belonging to the space $B C\left(\mathbb{R}_{+}, \mathbb{R}\right)$ are fixed points of the operator $Q$.

Proof. Observe that for any function $x \in B C\left(\mathbb{R}_{+}, \mathbb{R}\right), G x$ and $F x$ are continuous on $\mathbb{R}_{+}$. We show that the same holds also for $U x$. Take an arbitrary function $x \in B C\left(\mathbb{R}_{+}, \mathbb{R}\right)$ and fix $T>$ $0, \varepsilon>0$. Next assume that $t_{1}, t_{2} \in[0, T]$ such that $\left|t_{2}-t_{1}\right|<\varepsilon$. Without loss of generality one can assume that $t_{1}<t_{2}$. Then, in view of imposed assumptions, one has

$$
\begin{aligned}
& \left|(U x)\left(t_{2}\right)-(U x)\left(t_{1}\right)\right| \\
& =\frac{1}{\Gamma(\alpha)} \mid \int_{0}^{t_{1}}\left(t_{2}-s\right)^{\alpha-1} f_{3}\left(t_{2}, s, x\left(\gamma_{1}(s)\right), x\left(\gamma_{2}(s)\right)\right) \mathrm{d} s \\
& +\int_{t_{1}}^{t_{2}}\left(t_{2}-s\right)^{\alpha-1} f_{3}\left(t_{2}, s, x\left(\gamma_{1}(s)\right), x\left(\gamma_{2}(s)\right)\right) \mathrm{d} s \\
& -\int_{0}^{t_{1}}\left(t_{1}-s\right)^{\alpha-1} f_{3}\left(t_{1}, s, x\left(\gamma_{1}(s)\right), x\left(\gamma_{2}(s)\right)\right) \mathrm{d} s \mid \\
& \leq \frac{1}{\Gamma(\alpha)} \int_{0}^{t_{1}} \mid\left(t_{2}-s\right)^{\alpha-1} f_{3}\left(t_{2}, s, x\left(\gamma_{1}(s)\right), x\left(\gamma_{2}(s)\right)\right) \\
& -\left(t_{2}-s\right)^{\alpha-1} f_{3}\left(t_{1}, s, x\left(\gamma_{1}(s)\right), x\left(\gamma_{2}(s)\right)\right) \mid \mathrm{d} s \\
& +\frac{1}{\Gamma(\alpha)} \int_{0}^{t_{1}} \mid\left(t_{2}-s\right)^{\alpha-1} f_{3}\left(t_{1}, s, x\left(\gamma_{1}(s)\right), x\left(\gamma_{2}(s)\right)\right) \\
& -\left(t_{1}-s\right)^{\alpha-1} f_{3}\left(t_{1}, s, x\left(\gamma_{1}(s)\right), x\left(\gamma_{2}(s)\right)\right) \mid \mathrm{d} s \\
& +\frac{1}{\Gamma(\alpha)} \int_{t_{1}}^{t_{2}}\left|\left(t_{2}-s\right)^{\alpha-1} f_{3}\left(t_{2}, s, x\left(\gamma_{1}(s)\right), x\left(\gamma_{2}(s)\right)\right)\right| \mathrm{d} s \\
& \leq \frac{1}{\Gamma(\alpha)} \int_{0}^{t_{1}}\left(t_{2}-s\right)^{\alpha-1} \mid f_{3}\left(t_{2}, s, x\left(\gamma_{1}(s)\right), x\left(\gamma_{2}(s)\right)\right) \\
& -f_{3}\left(t_{1}, s, x\left(\gamma_{1}(s)\right), x\left(\gamma_{2}(s)\right)\right) \mid \mathrm{d} s \\
& +\frac{1}{\Gamma(\alpha)} \int_{0}^{t_{1}}\left|f_{3}\left(t_{1}, s, x\left(\gamma_{1}(s)\right), x\left(\gamma_{2}(s)\right)\right)\right| \\
& \times\left[\left(t_{1}-s\right)^{\alpha-1}-\left(t_{2}-s\right)^{\alpha-1}\right] \mathrm{d} s
\end{aligned}
$$

$$
\begin{aligned}
& +\frac{1}{\Gamma(\alpha)} \int_{t_{1}}^{t_{2}}\left(t_{2}-s\right)^{\alpha-1}\left|f_{3}\left(t_{2}, s, x\left(\gamma_{1}(s)\right), x\left(\gamma_{2}(s)\right)\right)\right| \mathrm{d} s \\
\leq & \frac{1}{\Gamma(\alpha)} \int_{0}^{t_{1}} \omega_{1}^{T}\left(f_{3}, \varepsilon,\|x\|\right)\left(t_{2}-s\right)^{\alpha-1} \mathrm{~d} s \\
& +\frac{1}{\Gamma(\alpha)} \int_{0}^{t_{1}}\left(\mid f_{3}\left(t_{1}, s, x\left(\gamma_{1}(s)\right), x\left(\gamma_{2}(s)\right)\right)\right. \\
& \left.-f_{3}\left(t_{1}, s, 0,0\right) \mid+F_{3}\left(t_{1}\right)\right) \\
& +\frac{1}{\Gamma(\alpha)} \int_{t_{1}}^{t_{2}}\left(t_{2}-s\right)^{\alpha-1}\left(\mid f_{3}\left(t_{2}, s, x\left(\gamma_{1}(s)\right), x\left(\gamma_{2}(s)\right)\right)\right. \\
\leq & \frac{\omega_{1}^{T}\left(f_{3}, \varepsilon,\|x\|\right)}{\Gamma(\alpha)} \frac{t_{2}^{\alpha}-\left(t_{2}-t_{1}\right)^{\alpha}}{\alpha}+\frac{1}{\Gamma(\alpha)} \\
& \times \int_{0}^{t_{1}}\left[n\left(t_{1}\right) \varphi(2\|x\|)+F_{3}\left(t_{1}\right)\right] \\
\leq & \frac{1}{\Gamma(\alpha+1)}\left\{t_{1}^{\alpha} \omega_{1}^{T}\left(f_{3}, \varepsilon,\|x\|\right)+\left(t_{2}-t_{1}\right)\right) \mathrm{d} s \\
& \times\left[\left(t_{1}-s\right)^{\alpha-1}-\left(t_{2}-s\right)^{\alpha-1}\right] \mathrm{d} s \\
& +\frac{1}{\Gamma(\alpha)} \int_{t_{1}}^{t_{2}}\left(t_{2}-s\right)^{\alpha-1}\left[n\left(t_{2}\right) \varphi(2\|x\|)+F_{3}\left(t_{2}\right)\right] \mathrm{d} s \\
\leq & \frac{\omega_{1}^{T}\left(f_{3}, \varepsilon,\|x\|\right)}{\Gamma(\alpha+1)} t_{1}^{\alpha}+\frac{n\left(t_{1}\right) \varphi(2\|x\|)+F_{3}\left(t_{1}\right)}{\Gamma(\alpha+1)} \\
& \times\left[t_{1}^{\alpha}-t_{2}^{\alpha}+\left(t_{2}-t_{1}\right)^{\alpha}\right] \\
& +(2\|x\|)+F_{3}\left(t_{2}\right) \\
& \\
&
\end{aligned}
$$

where

$$
\begin{aligned}
& \omega_{1}^{T}\left(f_{3}, \varepsilon,\|x\|\right) \\
& =\sup \left\{\left|f_{3}\left(t_{2}, s, y_{1}, y_{2}\right)-f_{3}\left(t_{1}, s, y_{1}, y_{2}\right)\right|: s, t_{1}, t_{2} \in[0, T],\right. \\
& \left.\quad s \leq t_{1}, s \leq t_{2},\left|t_{2}-t_{1}\right| \leq \varepsilon,\left|y_{1}\right| \leq\|x\|,\left|y_{2}\right| \leq\|x\|\right\} .
\end{aligned}
$$

Obviously, in view of the uniform continuity of $f_{3}\left(t, s, y_{1}, y_{2}\right)$ on the set $[0, T] \times[0, T] \times[-\|x\|,\|x\|] \times[-\|x\|,\|x\|]$, one has that $\omega_{1}^{T}\left(f_{3}, \varepsilon,\|x\|\right) \rightarrow 0$ as $\varepsilon \rightarrow 0$. In what follows, denote

$$
\begin{aligned}
& \bar{n}(T)=\max \{n(t): t \in[0, T]\}, \\
& \bar{F}_{3}(T)=\max \left\{F_{3}(t): t \in[0, T]\right\} .
\end{aligned}
$$


Then, keeping in mind the estimate (37) one obtains

$$
\begin{aligned}
& \left|(U x)\left(t_{2}\right)-(U x)\left(t_{1}\right)\right| \\
& \leq \frac{1}{\Gamma(\alpha+1)}\left\{T^{\alpha} \omega_{1}^{T}\left(f_{3}, \varepsilon,\|x\|\right)\right. \\
& \left.+2 \varepsilon^{\alpha}\left[\bar{n}(T) \varphi(2\|x\|)+\bar{F}_{3}(T)\right]\right\} .
\end{aligned}
$$

From the above inequality one can infer that the function $U x$ is continuous on the interval $[0, T]$ for any $T>0$. This yields the continuity of $U x$ on $\mathbb{R}_{+}$.

Finally, combining the continuity of the functions $G x, F x$, and $U x$, one deduces that the function $Q x$ is continuous on on $\mathbb{R}_{+}$.

Now, taking an arbitrary function $x \in B C\left(\mathbb{R}_{+}, \mathbb{R}\right)$, then, using our assumptions, for a fixed $t \in \mathbb{R}_{+}$, one has

$$
\begin{aligned}
& |Q x(t)| \\
& \leq|q(t)|+\left|f_{1}\left(t, x\left(\alpha_{1}(t)\right), x\left(\alpha_{2}(t)\right)\right)\right| \\
& +\frac{\left|f_{2}\left(t, x\left(\beta_{1}(t)\right), x\left(\beta_{2}(t)\right)\right)\right|}{\Gamma(\alpha)} \\
& \times \int_{0}^{t}(t-s)^{\alpha-1}\left|f_{3}\left(t, s, x\left(\gamma_{1}(s)\right), x\left(\gamma_{2}(s)\right)\right)\right| \mathrm{d} s \\
& \leq|q(t)|+\left|f_{1}\left(t, x\left(\alpha_{1}(t)\right), x\left(\alpha_{2}(t)\right)\right)-F_{1}(t)\right|+F_{1} \\
& +\frac{1}{\Gamma(\alpha)}\left[\left|f_{2}\left(t, x\left(\beta_{1}(t)\right),\left(\beta_{2}(t)\right)\right)-F_{2}(t)\right|+\left|F_{2}(t)\right|\right] \\
& \times \int_{0}^{t}(t-s)^{\alpha-1}\left[\mid f_{3}\left(t, s, x\left(\gamma_{1}(s)\right), x\left(\gamma_{2}(s)\right)\right)\right. \\
& \left.-f_{3}(t, s, 0,0) \mid+F_{3}(t)\right] \mathrm{d} s \\
& \leq\|q\|+\frac{\ell(t) \max \left\{\left|x\left(\alpha_{1}(t)\right)\right|,\left|x\left(\alpha_{2}(t)\right)\right|\right\}}{M+\max \left\{\left|x\left(\alpha_{1}(t)\right)\right|,\left|x\left(\alpha_{2}(t)\right)\right|\right\}}+F_{1} \\
& +\frac{2\|x\| m(t)+\left|F_{2}(t)\right|}{\Gamma(\alpha)} \\
& \times \int_{0}^{t}(t-s)^{\alpha-1}\left[n(t) \varphi(2\|x\|)+F_{3}(t)\right] \mathrm{d} s \\
& \leq\|q\|+\frac{L\|x\|}{M+\|x\|}+F_{1}+\frac{2\|x\| m(t)+\left|F_{2}(t)\right|}{\Gamma(\alpha)} \\
& \times\left[n(t) \varphi(2\|x\|)+F_{3}(t)\right] \int_{0}^{t}(t-s)^{\alpha-1} \mathrm{~d} s \\
& \leq\|q\|+\frac{L\|x\|}{M+\|x\|}+F_{1} \\
& +\left(t^{\alpha}[2 m(t) n(t)\|x\| \varphi(2\|x\|)\right. \\
& +2\|x\| m(t) F_{3}(t)+\left|F_{2}(t)\right| n(t) \varphi(2\|x\|) \\
& \left.\left.+\left|F_{2}(t)\right| F_{3}(t)\right]\right) \times(\Gamma(\alpha+1))^{-1}
\end{aligned}
$$

$$
\begin{aligned}
\leq & \|q\|+\frac{L\|x\|}{M+\|x\|}+F_{1} \\
& +\frac{a(t)\|x\| \varphi(2\|x\|)+b(t)\|x\|+c(t) \varphi(2\|x\|)+d(t)}{\Gamma(\alpha+1)} .
\end{aligned}
$$

Hence, in view of assumption $\left(H_{6}\right)$ one can infer that the function $Q x$ is bounded on $\mathbb{R}_{+}$. This assertion in conjunction with the continuity of $Q x$ on $\mathbb{R}_{+}$allows us to conclude that $Q x \in B C\left(\mathbb{R}_{+}, \mathbb{R}\right)$. Moreover, from the estimate (41) one obtains

$$
\begin{aligned}
|Q x(t)| \leq & \|q\|+\frac{L\|x\|}{M+\|x\|} \\
& +F_{1}+\frac{A\|x\| \varphi(2\|x\|)+B\|x\|+C \varphi(2\|x\|)+D}{\Gamma(\alpha+1)} .
\end{aligned}
$$

Linking this estimate with assumption $\left(\mathrm{H}_{7}\right)$, one deduces that there exists $r_{0}>0$ such that the operator $Q$ transforms the ball $B_{r_{0}}$ into itself.

Finally, let us notice that the second assertion of our lemma is obvious in the light of the fact that the operator $Q$ transforms the space $B C\left(\mathbb{R}_{+}, \mathbb{R}\right)$ into itself. The proof is complete.

Now, we are prepared to state and prove our main theorem of this section.

Theorem 19. Under the above assumptions $\left(H_{0}\right)-\left(H_{7}\right),(21)$ has at least one solution in the space $B C\left(\mathbb{R}_{+}, \mathbb{R}\right)$. Moreover, these solutions are globally uniformly attractive on $\mathbb{R}_{+}$.

Proof. In what follows we will consider the operator $Q$ as a mapping from $B_{r_{0}}$ into itself. Now we show that the operator $Q$ is continuous on the ball $B_{r_{0}}$. To do this, fix arbitrarily $\varepsilon>0$ and take $x, y \in B_{r_{0}}$ such that $\|x-y\|<\varepsilon$. Then one gets

$$
\begin{aligned}
& |Q x(t)-Q y(t)| \\
& =\mid f_{1}\left(t, x\left(\alpha_{1}(t)\right), x\left(\alpha_{2}(t)\right)\right) \\
& -f_{1}\left(t, y\left(\alpha_{1}(t)\right), y\left(\alpha_{2}(t)\right)\right) \\
& +\mid \frac{f_{2}\left(t, x\left(\beta_{1}(t)\right), x\left(\beta_{2}(t)\right)\right)}{\Gamma(\alpha)} \\
& \times \int_{0}^{t}(t-s)^{\alpha-1} f_{3}\left(t, s, x\left(\gamma_{1}(s)\right), x\left(\gamma_{2}(s)\right)\right) \mathrm{d} s \\
& -\frac{f_{2}\left(t, y\left(\beta_{1}(t)\right), y\left(\beta_{2}(t)\right)\right)}{\Gamma(\alpha)} \\
& \times \int_{0}^{t}(t-s)^{\alpha-1} f_{3}\left(t, s, y\left(\gamma_{1}(s)\right), y\left(\gamma_{2}(s)\right)\right) \mathrm{d} s \\
& \leq \mid f_{1}\left(t, x\left(\alpha_{1}(t)\right), x\left(\alpha_{2}(t)\right)\right) \\
& -f_{1}\left(t, y\left(\alpha_{1}(t)\right), y\left(\alpha_{2}(t)\right)\right)
\end{aligned}
$$




$$
\begin{aligned}
& +\left(\mid f_{2}\left(t, x\left(\beta_{1}(t)\right), x\left(\beta_{2}(t)\right)\right)\right. \\
& \left.-f_{2}\left(t, y\left(\beta_{1}(t)\right), y\left(\beta_{2}(t)\right)\right) \mid\right) \times(\Gamma(\alpha))^{-1} \\
& \times \int_{0}^{t} \frac{\left|f_{3}\left(t, s, x\left(\gamma_{1}(s)\right), x\left(\gamma_{2}(s)\right)\right)\right|}{(t-s)^{1-\alpha}} \mathrm{d} s \\
& +\frac{\left|f_{2}\left(t, y\left(\beta_{1}(t)\right), y\left(\beta_{2}(t)\right)\right)\right|}{\Gamma(\alpha)} \\
& \times \int_{0}^{t}\left(\left(\mid f_{3}\left(t, s, x\left(\gamma_{1}(s)\right), x\left(\gamma_{2}(s)\right)\right)\right.\right. \\
& \left.-f_{3}\left(t, s, y\left(\gamma_{1}(s)\right), y\left(\gamma_{2}(s)\right)\right) \mid\right) \\
& \left.\times\left((t-s)^{1-\alpha}\right)^{-1}\right) \mathrm{d} s \\
& \leq\left(\ell ( t ) \operatorname { m a x } \left\{\left|x\left(\alpha_{1}(t)\right)-y\left(\alpha_{1}(t)\right)\right|,\right.\right. \\
& \left.\left.\left|x\left(\alpha_{2}(t)\right)-y\left(\alpha_{2}(t)\right)\right|\right\}\right) \\
& \times\left(M+\max \left\{\left|x\left(\alpha_{1}(t)\right)-y\left(\alpha_{1}(t)\right)\right|,\right.\right. \\
& \left.\left.\left|x\left(\alpha_{2}(t)\right)-y\left(\alpha_{2}(t)\right)\right|\right\}\right)^{-1} \\
& +\left(m ( t ) \left(\left|x\left(\beta_{1}(t)\right)-y\left(\beta_{1}(t)\right)\right|\right.\right. \\
& \left.\left.+\left|x\left(\beta_{2}(t)\right)-y\left(\beta_{2}(t)\right)\right|\right)\right) \times(\Gamma(\alpha))^{-1} \\
& \times \int_{0}^{t}\left(\left(\left|f_{3}\left(t, s, x\left(\gamma_{1}(s)\right), x\left(\gamma_{2}(s)\right)\right)-f_{3}(t, s, 0,0)\right|\right.\right. \\
& \left.\left.+F_{3}(t)\right) \times\left((t-s)^{1-\alpha}\right)^{-1}\right) \mathrm{d} s \\
& +\frac{\left|f_{2}\left(t, y\left(\beta_{1}(t)\right), y\left(\beta_{2}(t)\right)\right)-F_{2}(t)\right|+\left|F_{2}(t)\right|}{\Gamma(\alpha)} \\
& \times \int_{0}^{t}\left(n ( t ) \varphi \left(\left|x\left(\gamma_{1}(s)\right)-y\left(\gamma_{1}(s)\right)\right|\right.\right. \\
& \left.\left.+\left|x\left(\gamma_{2}(s)\right)-y\left(\gamma_{2}(s)\right)\right|\right) \times\left((t-s)^{1-\alpha}\right)^{-1}\right) \mathrm{d} s \\
& \leq \frac{L\|x-y\|}{M+\|x-y\|}+\frac{2 m(t)\|x-y\|}{\Gamma(\alpha)} \\
& \times\left[n(t) \varphi(2\|x\|)+F_{3}(t)\right] \int_{0}^{t}(t-s)^{\alpha-1} \mathrm{~d} s \\
& +\frac{2 m(t)\|y\|+\left|F_{2}(t)\right|}{\Gamma(\alpha)} n(t) \varphi(2\|x-y\|) \\
& \times \int_{0}^{t}(t-s)^{\alpha-1} \mathrm{~d} s
\end{aligned}
$$

Now, linking the above established facts one concludes that the operator $Q$ maps continuously the closed ball $B_{r_{0}}$ into itself.

Further, taking a nonempty subset $X$ of the ball $B_{r_{0}}$, fixing arbitrarily $T>0$ and $\varepsilon>0$ and choosing $x \in X$ and $t_{1}, t_{2} \in[0, T]$ with $\left|t_{2}-t_{2}\right|<\varepsilon$, without loss of generality we may assume that $t_{1}<t_{2}$. Then, taking into account our assumptions $\left(\mathrm{H}_{2}\right)$ and $\left(\mathrm{H}_{4}\right)$, one gets

$$
\begin{aligned}
& \left|(Q x)\left(t_{2}\right)-(Q x)\left(t_{1}\right)\right| \\
& \leq\left|q\left(t_{1}\right)-q\left(t_{2}\right)\right| \\
& +\mid f_{1}\left(t_{2}, x\left(\alpha_{1}\left(t_{2}\right)\right),\left(\alpha_{2}\left(t_{2}\right)\right)\right) \\
& -f_{1}\left(t_{2}, x\left(\alpha_{1}\left(t_{1}\right)\right),\left(\alpha_{2}\left(t_{1}\right)\right)\right) \\
& +\mid f_{1}\left(t_{2}, x\left(\alpha_{1}\left(t_{1}\right)\right),\left(\alpha_{2}\left(t_{1}\right)\right)\right) \\
& -f_{1}\left(t_{1}, x\left(\alpha_{1}\left(t_{1}\right)\right),\left(\alpha_{2}\left(t_{1}\right)\right)\right) \\
& +\left|(F x)\left(t_{2}\right)(U x)\left(t_{2}\right)-(F x)\left(t_{1}\right)(U x)\left(t_{2}\right)\right| \\
& +\left|(F x)\left(t_{1}\right)(U x)\left(t_{2}\right)-(F x)\left(t_{1}\right)(U x)\left(t_{1}\right)\right| \\
& \leq \omega^{T}(q, \varepsilon)+\left(\ell ( t ) \operatorname { m a x } \left\{\left|x\left(\alpha_{1}\left(t_{2}\right)\right)-x\left(\alpha_{1}\left(t_{1}\right)\right)\right|,\right.\right. \\
& \left.\left.\left|x\left(\alpha_{2}\left(t_{2}\right)\right)-x\left(\alpha_{2}\left(t_{1}\right)\right)\right|\right\}\right) \\
& \times\left(M+\max \left\{\left|x\left(\alpha_{1}\left(t_{2}\right)\right)-x\left(\alpha_{1}\left(t_{1}\right)\right)\right|,\right.\right. \\
& \left.\left.\left|x\left(\alpha_{2}\left(t_{2}\right)\right)-x\left(\alpha_{2}\left(t_{1}\right)\right)\right|\right\}\right)^{-1} \\
& +\omega_{1}^{T}\left(f_{1}, \varepsilon\right) \\
& +\left(\left(\mid f_{2}\left(t_{2}, x\left(\beta_{1}\left(t_{2}\right)\right), x\left(\beta_{2}\left(t_{2}\right)\right)\right)\right.\right. \\
& \left.\left.-f_{2}\left(t_{1}, x\left(\beta_{1}\left(t_{1}\right)\right), x\left(\beta_{2}\left(t_{1}\right)\right)\right) \mid\right) \times(\Gamma(\alpha))^{-1}\right) \\
& \times \int_{0}^{t_{2}} \frac{\left|f_{3}\left(t_{2}, s, x\left(\gamma_{1}(s)\right), x\left(\gamma_{2}(s)\right)\right)\right|}{\left(t_{2}-s\right)^{1-\alpha}} \mathrm{d} s \\
& +\frac{\left|f_{2}\left(t_{1}, x\left(\beta_{1}\left(t_{1}\right)\right), x\left(\beta_{2}\left(t_{1}\right)\right)\right)\right|}{\Gamma(\alpha+1)} \\
& \times\left\{T^{\alpha} \omega_{1}^{T}\left(f_{3}, \varepsilon, r_{0}\right)+2 \varepsilon^{\alpha}\left[\bar{n}(T) \varphi\left(2 r_{0}\right)+\bar{F}_{3}(T)\right]\right\} \\
& \leq \omega^{T}(q, \varepsilon) \\
& +\left(\ell ( t ) \operatorname { m a x } \left\{\left|x\left(\alpha_{1}\left(t_{2}\right)\right)-x\left(\alpha_{1}\left(t_{1}\right)\right)\right|,\right.\right. \\
& \left.\left.\left|x\left(\alpha_{2}\left(t_{2}\right)\right)-x\left(\alpha_{2}\left(t_{1}\right)\right)\right|\right\}\right) \\
& \times\left(M+\max \left\{\left|x\left(\alpha_{1}\left(t_{2}\right)\right)-x\left(\alpha_{1}\left(t_{1}\right)\right)\right|,\right.\right. \\
& \left.\left.\left|x\left(\alpha_{2}\left(t_{2}\right)\right)-x\left(\alpha_{2}\left(t_{1}\right)\right)\right|\right\}\right)^{-1} \\
& +\omega_{1}^{T}\left(f_{1}, \varepsilon\right) \\
& +\frac{1}{\Gamma(\alpha)}\left(\mid f_{2}\left(t_{2}, x\left(\beta_{1}\left(t_{2}\right)\right), x\left(\beta_{2}\left(t_{2}\right)\right)\right)\right. \\
& -f_{2}\left(t_{2}, x\left(\beta_{1}\left(t_{1}\right)\right), x\left(\beta_{2}\left(t_{1}\right)\right)\right) \\
& +\mid f_{2}\left(t_{2}, x\left(\beta_{1}\left(t_{1}\right)\right), x\left(\beta_{2}\left(t_{1}\right)\right)\right) \\
& \left.-f_{2}\left(t_{1}, x\left(\beta_{1}\left(t_{1}\right)\right), x\left(\beta_{2}\left(t_{1}\right)\right)\right) \mid\right)
\end{aligned}
$$




$$
\begin{aligned}
& \times \int_{0}^{t_{2}}\left(\left(\mid f_{3}\left(t_{2}, s, x\left(\gamma_{1}(s)\right), x\left(\gamma_{2}(s)\right)\right)\right.\right. \\
& \left.\left.-f_{3}\left(t_{2}, s, 0,0\right) \mid+F_{3}\left(t_{2}\right)\right) \times\left(\left(t_{2}-s\right)^{1-\alpha}\right)^{-1}\right) \mathrm{d} s \\
& +\frac{\left|f_{2}\left(t_{1}, x\left(\beta_{1}\left(t_{1}\right)\right), x\left(\beta_{2}\left(t_{1}\right)\right)\right)-F_{2}\left(t_{1}\right)\right|+\left|F_{2}\left(t_{1}\right)\right|}{\Gamma(\alpha+1)} \\
& \times\left\{T^{\alpha} \omega_{1}^{T}\left(f_{3}, \varepsilon, r_{0}\right)+2 \varepsilon^{\alpha}\left[\bar{n}(T) \varphi\left(2 r_{0}\right)+\bar{F}_{3}(T)\right]\right\} \\
& \leq \omega^{T}(q, \varepsilon) \\
& +\frac{L \max \left\{\omega^{T}\left(x, \omega^{T}\left(\alpha_{1}, \varepsilon\right)\right), \omega^{T}\left(x, \omega^{T}\left(\alpha_{2}, \varepsilon\right)\right)\right\}}{M+\max \left\{\omega^{T}\left(x, \omega^{T}\left(\alpha_{1}, \varepsilon\right)\right), \omega^{T}\left(x, \omega^{T}\left(\alpha_{2}, \varepsilon\right)\right)\right\}} \\
& +\omega_{1}^{T}\left(f_{1}, \varepsilon\right) \\
& +\left(m ( t _ { 2 } ) \left(\left|x\left(\beta_{1}\left(t_{2}\right)\right)-x\left(\beta_{1}\left(t_{1}\right)\right)\right|\right.\right. \\
& \left.\left.+\left|x\left(\beta_{2}\left(t_{2}\right)\right)-x\left(\beta_{2}\left(t_{2}\right)\right)\right|\right)\right) \times(\Gamma(\alpha))^{-1} \\
& +\omega_{1}^{T}\left(f_{2}, \varepsilon\right) \int_{0}^{t_{2}} \frac{n\left(t_{2}\right) \varphi(2\|x\|)+F_{3}\left(t_{2}\right)}{\left(t_{2}-s\right)^{1-\alpha}} \mathrm{d} s \\
& +\frac{m\left(t_{1}\right)\left(\left|x\left(\beta_{1}\left(t_{1}\right)\right)\right|+\left|x\left(\beta_{2}\left(t_{1}\right)\right)\right|\right)+\left|F_{2}\left(t_{1}\right)\right|}{\Gamma(\alpha+1)} \\
& \times\left\{T^{\alpha} \omega_{1}^{T}\left(f_{3}, \varepsilon, r_{0}\right)+2 \varepsilon^{\alpha}\left[\bar{n}(T) \varphi\left(2 r_{0}\right)+\bar{F}_{3}(T)\right]\right\} \\
& \leq \omega^{T}(q, \varepsilon) \\
& +\frac{L \max \left\{\omega^{T}\left(x, \omega^{T}\left(\alpha_{1}, \varepsilon\right)\right), \omega^{T}\left(x, \omega^{T}\left(\alpha_{2}, \varepsilon\right)\right)\right\}}{M+\max \left\{\omega^{T}\left(x, \omega^{T}\left(\alpha_{1}, \varepsilon\right)\right), \omega^{T}\left(x, \omega^{T}\left(\alpha_{2}, \varepsilon\right)\right)\right\}} \\
& +\omega_{1}^{T}\left(f_{1}, \varepsilon\right) \\
& +\frac{1}{\Gamma(\alpha+1)}\left[m ( t _ { 2 } ) \left[\omega^{T}\left(x, \omega^{T}\left(\beta_{1}, \varepsilon\right)\right)\right.\right. \\
& \left.+\omega^{T}\left(x, \omega^{T}\left(\beta_{2}, \varepsilon\right)\right)\right] \\
& \left.+\omega_{1}^{T}\left(f_{2}, \varepsilon\right)\right] t_{2}^{\alpha}\left[n\left(t_{2}\right) \varphi\left(2 r_{0}\right)+F_{3}\left(t_{2}\right)\right] \\
& +\frac{2 m\left(t_{1}\right) r_{0}+\left|F_{2}\left(t_{1}\right)\right|}{\Gamma(\alpha+1)} \\
& \times\left\{T^{\alpha} \omega_{1}^{T}\left(f_{3}, \varepsilon, r_{0}\right)+2 \varepsilon^{\alpha}\left[\bar{n}(T) \varphi\left(2 r_{0}\right)+\bar{F}_{3}(T)\right]\right\} \\
& \leq \omega^{T}(q, \varepsilon) \\
& +\frac{L \max \left\{\omega^{T}\left(x, \omega^{T}\left(\alpha_{1}, \varepsilon\right)\right), \omega^{T}\left(x, \omega^{T}\left(\alpha_{2}, \varepsilon\right)\right)\right\}}{M+\max \left\{\omega^{T}\left(x, \omega^{T}\left(\alpha_{1}, \varepsilon\right)\right), \omega^{T}\left(x, \omega^{T}\left(\alpha_{2}, \varepsilon\right)\right)\right\}} \\
& +\omega_{1}^{T}\left(f_{1}, \varepsilon\right) \\
& +\left(t_{2}^{\alpha}\left[m\left(t_{2}\right) n\left(t_{2}\right) \varphi\left(2 r_{0}\right)+m\left(t_{2}\right) F_{3}\left(t_{2}\right)\right]\right. \\
& \left.\times\left[\omega^{T}\left(x, \omega^{T}\left(\beta_{1}, \varepsilon\right)\right)+\omega^{T}\left(x, \omega^{T}\left(\beta_{2}, \varepsilon\right)\right)\right]\right) \\
& \times(\Gamma(\alpha+1))^{-1} \\
& +\frac{\omega_{1}^{T}\left(f_{2}, \varepsilon\right) t_{2}^{\alpha}\left[n\left(t_{2}\right) \varphi\left(2 r_{0}\right)+F_{3}\left(t_{2}\right)\right]}{\Gamma(\alpha+1)}
\end{aligned}
$$

$$
\begin{aligned}
+ & \frac{2 m\left(t_{1}\right) r_{0}+\left|F_{2}\left(t_{1}\right)\right|}{\Gamma(\alpha+1)} \\
\times & \left\{T^{\alpha} \omega_{1}^{T}\left(f_{3}, \varepsilon, r_{0}\right)+2 \varepsilon^{\alpha}\left[\bar{n}(T) \varphi\left(2 r_{0}\right)+\bar{F}_{3}(T)\right]\right\} \\
\leq & \omega^{T}(q, \varepsilon) \\
+ & \frac{L \max \left\{\omega^{T}\left(x, \omega^{T}\left(\alpha_{1}, \varepsilon\right)\right), \omega^{T}\left(x, \omega^{T}\left(\alpha_{2}, \varepsilon\right)\right)\right\}}{M+\max \left\{\omega^{T}\left(x, \omega^{T}\left(\alpha_{1}, \varepsilon\right)\right), \omega^{T}\left(x, \omega^{T}\left(\alpha_{2}, \varepsilon\right)\right)\right\}} \\
+ & \omega_{1}^{T}\left(f_{1}, \varepsilon\right) \\
+ & \left([ A \varphi ( 2 r _ { 0 } ) + B ] \left[\omega^{T}\left(x, \omega^{T}\left(\beta_{1}, \varepsilon\right)\right)\right.\right. \\
& \left.\left.+\omega^{T}\left(x, \omega^{T}\left(\beta_{2}, \varepsilon\right)\right)\right]\right) \times(2 \Gamma(\alpha+1))^{-1} \\
+ & \frac{\omega_{1}^{T}\left(f_{2}, \varepsilon\right) t_{2}^{\alpha}\left[n\left(t_{2}\right) \varphi\left(2 r_{0}\right)+F_{3}\left(t_{2}\right)\right]}{\Gamma(\alpha+1)} \\
+ & \frac{2 m\left(t_{1}\right) r_{0}+\left|F_{2}\left(t_{1}\right)\right|}{\Gamma(\alpha+1)} \\
\times & \left\{T^{\alpha} \omega_{1}^{T}\left(f_{3}, \varepsilon, r_{0}\right)+2 \varepsilon^{\alpha}\left[\bar{n}(T) \varphi\left(2 r_{0}\right)+\bar{F}_{3}(T)\right]\right\},
\end{aligned}
$$

where

$$
\begin{aligned}
& \omega^{T}(q, \varepsilon) \\
& =\sup \left\{q\left(t_{2}\right)-q\left(t_{1}\right): t_{1}, t_{2} \in[0, T],\left|t_{2}-t_{1}\right|<\varepsilon\right\}, \\
& \omega_{1}^{T}\left(f_{1}, \varepsilon\right) \\
& =\sup \left\{f_{1}\left(t_{2}, x, y\right)-f_{1}\left(t_{1}, x, y\right): t_{1}, t_{2} \in[0, T],\right. \\
& \left.\quad\left|t_{2}-t_{1}\right|<\varepsilon, x, y \in\left[-r_{0}, r_{0}\right]\right\}, \\
& \omega_{1}^{T}\left(f_{2}, \varepsilon\right) \\
& =\sup \left\{f_{2}\left(t_{2}, x, y\right)-f_{2}\left(t_{1}, x, y\right): t_{1}, t_{2} \in[0, T],\right. \\
& \left.\quad\left|t_{2}-t_{1}\right|<\varepsilon, x, y \in\left[-r_{0}, r_{0}\right]\right\} .
\end{aligned}
$$

Moreover, mention that other notations used in the above estimate were introduced earlier.

From the above estimate one can derive the following inequality:

$$
\begin{aligned}
& \omega^{T}(Q x, \varepsilon) \\
& \leq \omega^{T}(q, \varepsilon) \\
& \quad+\frac{L \max \left\{\omega^{T}\left(x, \omega^{T}\left(\alpha_{1}, \varepsilon\right)\right), \omega^{T}\left(x, \omega^{T}\left(\alpha_{2}, \varepsilon\right)\right)\right\}}{M+\max \left\{\omega^{T}\left(x, \omega^{T}\left(\alpha_{1}, \varepsilon\right)\right), \omega^{T}\left(x, \omega^{T}\left(\alpha_{2}, \varepsilon\right)\right)\right\}} \\
& +\omega_{r}^{T}\left(f_{1}, \varepsilon\right) \\
& +\frac{\left[A \varphi\left(2 r_{0}\right)+B\right]\left[\omega^{T}\left(x, \omega^{T}\left(\beta_{1}, \varepsilon\right)\right)+\omega^{T}\left(x, \omega^{T}\left(\beta_{2}, \varepsilon\right)\right)\right]}{2 \Gamma(\alpha+1)}
\end{aligned}
$$




$$
\begin{aligned}
& +\frac{\omega_{1}^{T}\left(f_{2}, \varepsilon\right) t_{2}^{\alpha}\left[n\left(t_{2}\right) \varphi\left(2 r_{0}\right)+F_{3}\left(t_{2}\right)\right]}{\Gamma(\alpha+1)} \\
& +\frac{2 m\left(t_{1}\right) r_{0}+\left|F_{2}\left(t_{1}\right)\right|}{\Gamma(\alpha+1)} \\
& \times\left\{T^{\alpha} \omega_{1}^{T}\left(f_{3}, \varepsilon, r_{0}\right)+2 \varepsilon^{\alpha}\left[\bar{n}(T) \varphi\left(2 r_{0}\right)+\bar{F}_{3}(T)\right]\right\} .
\end{aligned}
$$

Observe that $\omega^{T}(q, \varepsilon) \rightarrow 0, \omega_{1}^{T}\left(f_{1}, \varepsilon\right) \rightarrow 0, \omega_{1}^{T}\left(f_{2}, \varepsilon\right) \rightarrow 0$ and $\omega_{1}^{T}\left(f_{3}, \varepsilon\right) \rightarrow 0$ as $\varepsilon \rightarrow 0$, which is a simple consequence of the uniform continuity of the functions $q, f_{1}, f_{2}$, and $f_{3}$ on the sets $[0, T],[0, T] \times\left[-r_{0}, r_{0}\right] \times\left[-r_{0}, r_{0}\right],[0, T] \times$ $\left[-r_{0}, r_{0}\right] \times\left[-r_{0}, r_{0}\right]$ and $[0, T] \times[0, T] \times\left[-r_{0}, r_{0}\right] \times\left[-r_{0}, r_{0}\right]$, respectively. Moreover, it is obvious that the constant

$$
\begin{aligned}
\omega^{T}\left(\alpha_{1}, \varepsilon\right) & \longrightarrow 0, \quad \omega^{T}\left(\alpha_{2}, \varepsilon\right) \\
\omega^{T}\left(\beta_{1}, \varepsilon\right) & \longrightarrow 0, \quad \omega^{T}\left(\beta_{2}, \varepsilon\right) \\
& \longrightarrow 0, \\
\text { as } \varepsilon & \longrightarrow 0 .
\end{aligned}
$$

Thus, linking the established facts with the estimate (46) one gets

$$
\omega_{0}^{T}(Q X) \leq \frac{L \omega_{0}^{T}(X)}{M+\omega_{0}^{T}(X)} .
$$

Now, taking into account our assumptions, for arbitrarily fixed $T \in \mathbb{R}_{+}$as well as for $x_{1}, x_{2}, y_{1}, y_{2} \in X$ one can deduce the following estimate (cf. the estimate (41)-(44)):

$$
\begin{aligned}
& |Q x(t)-Q y(t)| \\
& \leq\left(L \operatorname { m a x } \left\{\left|x\left(\alpha_{1}(t)\right)-y\left(\alpha_{1}(t)\right)\right|,\right.\right. \\
& \left.\left.\left|x\left(\alpha_{2}(t)\right)-y\left(\alpha_{2}(t)\right)\right|\right\}\right) \\
& \times\left(M+\max \left\{\left|x\left(\alpha_{1}(t)\right)-y\left(\alpha_{1}(t)\right)\right|,\right.\right. \\
& \left.\left.\quad\left|x\left(\alpha_{2}(t)\right)-y\left(\alpha_{2}(t)\right)\right|\right\}\right)^{-1} \\
& +\left(m ( t ) \left(\left|x\left(\beta_{1}(t)\right)-y\left(\beta_{1}(t)\right)\right|\right.\right. \\
& \left.+\left|x\left(\beta_{2}(t)\right)-y\left(\beta_{2}(t)\right)\right|\right) \times(\Gamma(\alpha))^{-1} \\
& \times \int_{0}^{t}\left(\left(\left|f_{3}\left(t, s, x\left(\gamma_{1}(s)\right), x\left(\gamma_{2}(s)\right)\right)-f_{3}(t, s, 0,0)\right|\right.\right. \\
& \left.\left.+F_{3}(t)\right) \times\left((t-s)^{1-\alpha}\right)^{-1}\right) \mathrm{d} s \\
& +\frac{\left|f_{2}\left(t, y\left(\beta_{1}(t)\right), y\left(\beta_{2}(t)\right)\right)-F_{2}(t)\right|+\left|F_{2}(t)\right|}{\Gamma(\alpha)} \\
& \times \int_{0}^{t}\left(\left(n ( t ) \varphi \left(\left|x\left(\gamma_{1}(s)\right)-y\left(\gamma_{1}(s)\right)\right|\right.\right.\right. \\
& \left.\left.+\left|x\left(\gamma_{2}(s)\right)-y\left(\gamma_{2}(s)\right)\right|\right)\right) \\
& \left.\times\left((t-s)^{1-\alpha}\right)^{-1}\right) \mathrm{d} s
\end{aligned}
$$

$$
\begin{aligned}
& \leq \frac{L \max \left\{\operatorname{diam} X\left(\alpha_{1}(t)\right), \operatorname{diam} X\left(\alpha_{2}(t)\right)\right\}}{M+\max \left\{\operatorname{diam} X\left(\alpha_{1}(t)\right), \operatorname{diam} X\left(\alpha_{2}(t)\right)\right\}} \\
& +\frac{m(t)\left(\left|x\left(\beta_{1}(t)\right)-y\left(\beta_{1}(t)\right)\right|+\left|x\left(\beta_{2}(t)\right)-y\left(\beta_{2}(t)\right)\right|\right)}{\Gamma(\alpha)} \\
& \quad \times \int_{0}^{t} \frac{n(t) \varphi\left(\left|x\left(\gamma_{1}(s)\right)\right|+\left|x\left(\gamma_{2}(s)\right)\right|\right)+F_{3}(t)}{(t-s)^{1-\alpha}} \mathrm{d} s \\
& +\frac{m(t)\left(\left|y\left(\beta_{1}(t)\right)\right|+\left|y\left(\beta_{2}(t)\right)\right|\right)+\left|F_{2}(t)\right|}{\Gamma(\alpha)} \\
& \quad \times \int_{0}^{t}\left(n ( t ) \varphi \left(\left|x\left(\gamma_{1}(s)\right)-y\left(\gamma_{1}(s)\right)\right|\right.\right. \\
& \left.\quad+\left|x\left(\gamma_{2}(s)\right)-y\left(\gamma_{2}(s)\right)\right|\right) \\
& \left.\quad \times\left((t-s)^{1-\alpha}\right)^{-1}\right) \mathrm{d} s
\end{aligned}
$$$$
\leq \frac{L \max \left\{\operatorname{diam} X\left(\alpha_{1}(t)\right), \operatorname{diam} X\left(\alpha_{2}(t)\right)\right\}}{M+\max \left\{\operatorname{diam} X\left(\alpha_{1}(t)\right), \operatorname{diam} X\left(\alpha_{2}(t)\right)\right\}}
$$$$
+\left(m ( t ) n ( t ) \left(\left|x\left(\beta_{1}(t)\right)\right|+\left|y\left(\beta_{1}(t)\right)\right|\right.\right.
$$$$
\left.\left.+\left|x\left(\beta_{2}(t)\right)\right|+\left|y\left(\beta_{2}(t)\right)\right|\right)\right)
$$$$
\times(\Gamma(\alpha))^{-1}
$$$$
\times \int_{0}^{t} \frac{\varphi\left(\left|x\left(\gamma_{1}(s)\right)\right|+\left|x\left(\gamma_{2}(s)\right)\right|\right)}{(t-s)^{1-\alpha}} \mathrm{d} s
$$$$
+\left(m ( t ) F _ { 3 } ( t ) \left(\left|x\left(\beta_{1}(t)\right)\right|+\left|y\left(\beta_{1}(t)\right)\right|\right.\right.
$$$$
\left.\left.+\left|x\left(\beta_{2}(t)\right)\right|+\left|y\left(\beta_{2}(t)\right)\right|\right)\right)
$$$$
\times(\Gamma(\alpha))^{-1}
$$$$
\times \int_{0}^{t} \frac{\varphi\left(\left|x\left(\gamma_{1}(s)\right)\right|+\left|x\left(\gamma_{2}(s)\right)\right|\right)}{(t-s)^{1-\alpha}} \mathrm{d} s
$$$$
+\frac{m(t) n(t)\left(\left|y\left(\beta_{1}(t)\right)\right|+\left|y\left(\beta_{2}(t)\right)\right|\right)}{\Gamma(\alpha)}
$$$$
\times \int_{0}^{t}\left(\varphi \left(\left|x\left(\gamma_{1}(s)\right)\right|+\left|y\left(\gamma_{1}(s)\right)\right|\right.\right.
$$$$
\left.+\left|x\left(\gamma_{2}(s)\right)\right|+\left|y\left(\gamma_{2}(s)\right)\right|\right)
$$$$
\left.\times\left((t-s)^{1-\alpha}\right)^{-1}\right) \mathrm{d} s
$$$$
+\frac{n(t)\left|F_{2}(t)\right|\left(\left|y\left(\beta_{1}(t)\right)\right|+\left|y\left(\beta_{2}(t)\right)\right|\right)}{\Gamma(\alpha)}
$$$$
\times \int_{0}^{t}\left(\varphi \left(\left|x\left(\gamma_{1}(s)\right)\right|+\left|y\left(\gamma_{1}(s)\right)\right|\right.\right.
$$$$
\left.+\left|x\left(\gamma_{2}(s)\right)\right|+\left|y\left(\gamma_{2}(s)\right)\right|\right)
$$$$
\left.\times\left((t-s)^{1-\alpha}\right)^{-1}\right) \mathrm{d} s
$$$$
\leq \frac{L m a x}{M+\max \left\{\operatorname{diam} X\left(\alpha_{1}(t)\right), \operatorname{diam} X\left(\alpha_{1}(t)\right), \operatorname{diam} X\left(\alpha_{2}(t)\right)\right\}}
$$$$
+\frac{4 m(t) n(t) r_{0} \varphi\left(2 r_{0}\right)}{\Gamma(\alpha)} \int_{0}^{t}(t-s)^{\alpha-1} \mathrm{~d} s
$$ 


$$
\begin{aligned}
& +\frac{4 m(t) F_{3}(t) r_{0} \varphi\left(2 r_{0}\right)}{\Gamma(\alpha)} \int_{0}^{t}(t-s)^{\alpha-1} \mathrm{~d} s \\
& +\frac{2 m(t) n(t) r_{0} \varphi\left(4 r_{0}\right)}{\Gamma(\alpha)} \int_{0}^{t}(t-s)^{\alpha-1} \mathrm{~d} s \\
& +\frac{n(t)\left|F_{2}(t)\right| \varphi\left(4 r_{0}\right)}{\Gamma(\alpha)} \int_{0}^{t}(t-s)^{\alpha-1} \mathrm{~d} s \\
& =\frac{L \max \left\{\operatorname{diam} X\left(\alpha_{1}(t)\right), \operatorname{diam} X\left(\alpha_{2}(t)\right)\right\}}{M+\max \left\{\operatorname{diam} X\left(\alpha_{1}(t)\right), \operatorname{diam} X\left(\alpha_{2}(t)\right)\right\}} \\
& +\frac{2 a(t) r_{0} \varphi\left(2 r_{0}\right)}{\Gamma(\alpha+1)}+\frac{a(t) r_{0} \varphi\left(4 r_{0}\right)}{\Gamma(\alpha+1)} \\
& +\frac{c(t) \varphi\left(4 r_{0}\right)}{\Gamma(\alpha+1)}+\frac{2 b(t) r_{0} \varphi\left(2 r_{0}\right)}{\Gamma(\alpha+1)} .
\end{aligned}
$$

In view of assumptions $\left(H_{0}\right)$ and $\left(H_{6}\right)$ this yields

$$
\begin{aligned}
& \limsup _{t \rightarrow \infty} \operatorname{diam} Q X(t) \\
& \leq \frac{L \lim \sup _{t \rightarrow \infty} \max \left\{\operatorname{diam} X\left(\alpha_{1}(t)\right), \operatorname{diam} X\left(\alpha_{2}(t)\right)\right\}}{M+\lim \sup _{t \rightarrow \infty} \max \left\{\operatorname{diam} X\left(\alpha_{1}(t)\right), \operatorname{diam} X\left(\alpha_{2}(t)\right)\right\}} \\
& \leq \frac{L \lim \sup _{t \rightarrow \infty} \operatorname{diam} X(t)}{M+\lim \sup _{t \rightarrow \infty} \operatorname{diam} X(t)}
\end{aligned}
$$

Further, using the measure of noncompactness $\mu_{a}$ defined by the formula (9) and keeping in mind the estimates (48) and (50), one obtains

$$
\begin{aligned}
& \mu_{a}(Q X) \\
& =\max \left\{\omega_{0}(Q X), \limsup _{t \rightarrow \infty} \max \operatorname{diam}(Q X)\right\} \\
& \leq \max \left\{\frac{L \omega_{0}(X)}{M+\omega_{0}(X)}, \frac{L \lim \sup _{t \rightarrow \infty} \operatorname{diam} X(t)}{M+\limsup \operatorname{sum}_{t \rightarrow \infty} \operatorname{diam} X(t)}\right\} \\
& \leq \frac{L \max \left\{\omega_{0}(X), \limsup \sup _{t \rightarrow \infty} \operatorname{diam} X(t)\right\}}{M+\max \left\{\omega_{0}(X), \lim \sup _{t \rightarrow \infty} \operatorname{diam} X(t)\right\}} \\
& =\frac{L \mu_{a}(X)}{M+\mu_{a}(X)} .
\end{aligned}
$$

Since $L \leq M$ in view of assumption $\left(\mathrm{H}_{2}\right)$, from the above estimate one infers that $\mu_{a}(Q X) \leq \varphi\left(\mu_{a}(X)\right)$, where $\varphi(r)=$ $L r /(M+r)<r$ for $r>0$. Hence, apply Theorem 5 to deduce that the operator $Q$ has a fixed point $x$ in the ball $B_{r_{0}}$. On the other hand, from Remark 6 one concludes that the set $\operatorname{Fix}(Q)$ belongs to the family ker $\mu_{a}$. Now, taking into account the description of sets belonging to ker $\mu_{a}$ (given in Section 2) one deduces that all solutions for (21) are globally uniformly attractive on $\mathbb{R}_{+}$. This completes the proof.

Remark 20. When $q \equiv 0, f_{1}(t, x, y)=f_{1}(t, x), f_{2}(t, x, y)=$ $f_{2}(t, x)$ and $f_{3}(t, s, x, y)=f_{3}(t, s, x)$, according to our
Theorem 19, one can obtain the global attractivity result for (23) which has been studied by Banaś and O’Regan in [47]. Meanwhile when

$$
\begin{gathered}
q(t)=0, \\
f_{1}\left(t, x\left(\alpha_{1}(t)\right), x\left(\alpha_{2}(t)\right)\right)=g(t, x(\eta(t))), \\
f_{2}\left(t, x\left(\beta_{1}(t)\right), x\left(\beta_{2}(t)\right)\right)=f(t, x(\beta(t))), \\
f_{3}\left(t, s, x\left(\gamma_{1}(s)\right), x\left(\gamma_{2}(s)\right)\right)=h(t, s) u(s, x(\gamma(s))),
\end{gathered}
$$

from our Theorem 19, one can obtain the global attractivity result for (23) which has been studied by Balachandran et al. in [48].

To prove next result concerning the asymptotic positivity of the attractive solutions, we need the following hypothesis in the sequel.

$\left(H_{8}\right)$ The functions $q$ and $f_{1}$ satisfy $\lim _{t \rightarrow \infty}[|q(t)|-$ $q(t)]=0, \lim _{t \rightarrow \infty}\left[\left|f_{1}(t, x, y)\right|-f_{1}(t, x, y)\right]=0$ for all $x, y \in \mathbb{R}$.

Theorem 21. Under the hypotheses of Theorem 19 and $\left(H_{8}\right)$, (21) has at least one solution on $\mathbb{R}_{+}$. Moreover, these solutions are uniformly globally attractive and ultimately positive on $\mathbb{R}_{+}$.

Proof. Consider the closed ball $B_{r_{0}}$ in the Banach space $B C\left(\mathbb{R}_{+}, \mathbb{R}\right)$, where the real number $r_{0}$ is given as in the proof of Theorem 19, and define a mapping $Q: B C\left(\mathbb{R}_{+}, \mathbb{R}\right) \rightarrow$ $B C\left(\mathbb{R}_{+}, \mathbb{R}\right)$ by $(36)$. Then it is shown as in the proof of Theorem 19 that $Q$ defines a continuous mapping from the space $B C\left(\mathbb{R}_{+}, \mathbb{R}\right)$ into $B_{r_{0}}$. In particular, $Q$ maps $B_{r_{0}}$ into itself. Next we show that $Q$ is a nonlinear $D$-set-contraction with respect to the measure $\mu_{\text {ad }}$ of noncompactness in $B C\left(\mathbb{R}_{+}, \mathbb{R}\right)$. We know that, for any $x, y \in \mathbb{R}$, one has the inequality, $|x|+|y| \geq|x+y| \geq x+y$, and therefore,

$$
\begin{aligned}
|| x+y|-(x+y)| & \leq|| x|+| y|-(x+y)| \\
& \leq|| x|-x|+|| y|-y|
\end{aligned}
$$

for all $x, y \in \mathbb{R}$. Now for any $x \in B_{r_{0}}$, one has

$$
\begin{aligned}
& || Q x(t)|-Q x(t)| \\
& \leq|| q(t)|-q(t)| \\
& +|| f_{1}\left(t, x\left(\alpha_{1}(t)\right), x\left(\alpha_{2}(t)\right)\right) \mid \\
& -f_{1}\left(t, x\left(\alpha_{1}(t)\right), x\left(\alpha_{2}(t)\right)\right) \mid \\
& +\| \frac{f_{2}\left(t, x\left(\beta_{1}(t)\right), x\left(\beta_{2}(t)\right)\right)}{\Gamma(\alpha)} \\
& \times \int_{0}^{t}(t-s)^{\alpha-1} f_{3}\left(t, s, x\left(\gamma_{1}(s)\right), x\left(\gamma_{2}(s)\right)\right) \mathrm{d} s \mid \\
& -\frac{f_{2}\left(t, x\left(\beta_{1}(t)\right), x\left(\beta_{2}(t)\right)\right)}{\Gamma(\alpha)} \\
& \times \int_{0}^{t}(t-s)^{\alpha-1} f_{3}\left(t, s, x\left(\gamma_{1}(s)\right), x\left(\gamma_{2}(s)\right)\right) \mathrm{d} s \mid
\end{aligned}
$$




$$
\begin{aligned}
& \leq \delta_{T}(q)+\delta_{T}\left(f_{1}\right) \\
& +2 \mid \frac{f_{2}\left(t, x\left(\beta_{1}(t)\right), x\left(\beta_{2}(t)\right)\right)}{\Gamma(\alpha)} \\
& \quad \times \int_{0}^{t}(t-s)^{\alpha-1} f_{3}\left(t, s, x\left(\gamma_{1}(s)\right), x\left(\gamma_{2}(s)\right)\right) \mathrm{d} s \mid \\
& \leq \delta_{T}(q)+\delta_{T}\left(f_{1}\right) \\
& +(2[a(t)\|x\| \varphi(2\|x\|)+b(t)\|x\| \\
& \quad+c(t) \varphi(2\|x\|)+d(t)]) \times(\Gamma(\alpha+1))^{-1} .
\end{aligned}
$$

From the above inequality, it follows that

$$
\begin{aligned}
& \delta_{T}(X) \\
& \leq \delta_{T}(q)+\delta_{T}\left(f_{1}\right) \\
& +(2[a(t)\|x\| \varphi(2\|x\|)+b(t)\|x\| \\
& \quad+c(t) \varphi(2\|x\|)+d(t)]) \times(\Gamma(\alpha+1))^{-1} .
\end{aligned}
$$

For all closed $X \subset B_{r_{0}}$. Taking the limit superior as $T \rightarrow \infty$, one obtains

$$
\underset{T \rightarrow \infty}{\limsup } \delta_{T}(X) \leq \limsup _{T \rightarrow \infty} \delta_{T}(q)+\limsup _{T \rightarrow \infty} \delta_{T}\left(f_{1}\right)
$$

for all closed $X \subset B_{r_{0}}$. Hence $\delta(Q X)=\lim _{T \rightarrow \infty} \delta_{T}(X)=0$ for all closed subsets $X$ of $B_{r_{0}}$. Further, using the measure of noncompactness $\mu_{a}$ defined by the formula (9) and keeping in mind the estimates (50) and (51), one obtains

$$
\begin{aligned}
\mu_{\mathrm{ad}}(Q X) & =\max \left\{\mu_{a}(Q X), \delta(Q X)\right\} \\
& \leq \max \left\{\frac{L \mu_{a}(X)}{M+\mu_{a}(X)}, 0\right\} \\
& \leq \frac{L \max \left\{\mu_{a}(X), 0\right\}}{M+\max \left\{\mu_{a}(X), 0\right\}} \leq \frac{L \mu_{\mathrm{ad}}(X)}{M+\mu_{\mathrm{ad}}(X)} .
\end{aligned}
$$

Since $L \leq M$ in view of assumption $\left(\mathrm{H}_{2}\right)$, from the above estimate one infers that $\mu_{\mathrm{ad}}(Q X) \leq \varphi\left(\mu_{\mathrm{ad}}(Q X)\right)$, where $\varphi(r)=$ $\mathrm{Lr} /(M+r)<r$ for $r>0$. Hence, applying Theorem 5 to deduce that the operator $Q$ has a fixed point $x$ in the ball $B_{r_{0}}$. Obviously $x$ is a solution of (21). Now, taking into account the description of sets belonging to ker $\mu_{\text {ad }}$ (given in Section 2) one deduces that all solutions of (21) are uniformly globally attractive and ultimately positive on $\mathbb{R}_{+}$. This completes the proof.

Next we prove the global asymptotic attractivity results for (21). We need the following hypotheses in the sequel.

$\left(H_{9}\right)$ The function $q: \mathbb{R}_{+} \rightarrow \mathbb{R}$ is continuous and $\lim _{t \rightarrow \infty} q(t)=c$.

$\left(H_{10}\right) f_{1}(t, 0,0)=0$ for all $t \in \mathbb{R}_{+}$, and

$\left(H_{11}\right) \lim _{t \rightarrow \infty} \ell(t)=0$, where the function $\ell$ is defined as in hypothesis $\left(\mathrm{H}_{2}\right)$.
Theorem 22. Assume that the hypotheses $\left(\mathrm{H}_{0}\right),\left(\mathrm{H}_{2}\right)-\left(\mathrm{H}_{7}\right)$ and $\left(H_{9}\right)-\left(H_{11}\right)$ hold. Then $(21)$ has at least one solution in the space $B C\left(\mathbb{R}_{+}, \mathbb{R}\right)$. Moreover, these solutions are uniformly globally asymptotically attractive on $\mathbb{R}_{+}$.

Proof. Consider the closed ball $B_{r_{0}}$ in the Banach space $B C\left(\mathbb{R}_{+}, \mathbb{R}\right)$, where the real number $r_{0}$ is given as in the proof of Theorem 19 and define a mapping $Q: B_{r_{0}} \rightarrow B_{r_{0}}$ by (36). Then $Q$ is continuous and maps the space $B C\left(\mathbb{R}^{+}, \mathbb{R}\right)$ and, in particular, $B_{r_{0}}$ into $B_{r_{0}}$. We show that $Q$ is a nonlinear $D$-set-contraction with respect to the measure $\mu_{c}$ of noncompactness in $B C\left(\mathbb{R}_{+}, \mathbb{R}\right)$. Let $x \in B_{r_{0}}$ be arbitrary. Then one has

$|Q x(t)-c|$

$$
\begin{aligned}
& \leq|q(t)-c|+\left|f_{1}\left(t, x\left(\alpha_{1}(t)\right), x\left(\alpha_{2}(t)\right)\right)\right| \\
& +\mid \frac{f_{2}\left(t, x\left(\beta_{1}(t)\right), x\left(\beta_{2}(t)\right)\right)}{\Gamma(\alpha)} \\
& \quad \times \int_{0}^{t}(t-s)^{\alpha-1} f_{3}\left(t, s, x\left(\gamma_{1}(s)\right), x\left(\gamma_{2}(s)\right)\right) \mathrm{d} s \mid \\
& \leq|q(t)-c|+\left|f_{1}\left(t, x\left(\alpha_{1}(t)\right), x\left(\alpha_{2}(t)\right)\right)\right| \\
& +\frac{\left|f_{2}\left(t, x\left(\beta_{1}(t)\right), x\left(\beta_{2}(t)\right)\right)\right|}{\Gamma(\alpha)} \\
& \quad \times \int_{0}^{t}(t-s)^{\alpha-1}\left|f_{3}\left(t, s, x\left(\gamma_{1}(s)\right), x\left(\gamma_{2}(s)\right)\right)\right| \mathrm{d} s
\end{aligned}
$$$$
\leq|q(t)-c|+\left|f_{1}\left(t, x\left(\alpha_{1}(t)\right), x\left(\alpha_{2}(t)\right)\right)-F_{1}(t)\right|
$$$$
+\frac{1}{\Gamma(\alpha)}\left[\left|f_{2}\left(t, x\left(\beta_{1}(t)\right),\left(\beta_{2}(t)\right)\right)-F_{2}(t)\right|+\left|F_{2}(t)\right|\right]
$$$$
\times \int_{0}^{t}(t-s)^{\alpha-1}\left[\mid f_{3}\left(t, s, x\left(\gamma_{1}(s)\right), x\left(\gamma_{2}(s)\right)\right)\right.
$$$$
\left.-f_{3}(t, s, 0,0) \mid+F_{3}(t)\right] \mathrm{d} s
$$

$$
\leq|q(t)-c|+\frac{\ell(t) \max \left\{\left|x\left(\alpha_{1}(t)\right)\right|,\left|x\left(\alpha_{2}(t)\right)\right|\right\}}{M+\max \left\{\left|x\left(\alpha_{1}(t)\right)\right|,\left|x\left(\alpha_{2}(t)\right)\right|\right\}}
$$$$
+\frac{2\|x\| m(t)+\left|F_{2}(t)\right|}{\Gamma(\alpha)}
$$$$
\times \int_{0}^{t}(t-s)^{\alpha-1}\left[n(t) \varphi(2\|x\|)+F_{3}(t)\right] \mathrm{d} s
$$$$
\leq|q(t)-c|+\frac{\ell(t) \max \left\{\left|x\left(\alpha_{1}(t)\right)\right|,\left|x\left(\alpha_{2}(t)\right)\right|\right\}}{M+\max \left\{\left|x\left(\alpha_{1}(t)\right)\right|,\left|x\left(\alpha_{2}(t)\right)\right|\right\}}
$$

$$
+\frac{2\|x\| m(t)+\left|F_{2}(t)\right|}{\Gamma(\alpha)}\left[n(t) \varphi(2\|x\|)+F_{3}(t)\right]
$$$$
\times \int_{0}^{t}(t-s)^{\alpha-1} \mathrm{~d} s
$$

$$
\leq|q(t)-c|+\frac{\ell(t) \max \left\{\left|x\left(\alpha_{1}(t)\right)\right|,\left|x\left(\alpha_{2}(t)\right)\right|\right\}}{M+\max \left\{\left|x\left(\alpha_{1}(t)\right)\right|,\left|x\left(\alpha_{2}(t)\right)\right|\right\}}
$$




$$
\begin{aligned}
& +\left(t ^ { \alpha } \left[2 m(t) n(t)\|x\| \varphi(2\|x\|)+2\|x\| m(t) F_{3}(t)\right.\right. \\
& +\left|F_{2}(t)\right| n(t) \varphi(2\|x\|) \\
& \left.\left.+\left|F_{2}(t)\right| F_{3}(t)\right]\right) \times(\Gamma(\alpha+1))^{-1} \\
& \leq|q(t)-c|+\frac{\ell(t) \max \left\{\left|x\left(\alpha_{1}(t)\right)\right|,\left|x\left(\alpha_{2}(t)\right)\right|\right\}}{M+\max \left\{\left|x\left(\alpha_{1}(t)\right)\right|,\left|x\left(\alpha_{2}(t)\right)\right|\right\}} \\
& +\frac{a(t)\|x\| \varphi(2\|x\|)+b(t)\|x\|+c(t) \varphi(2\|x\|)+d(t)}{\Gamma(\alpha+1)} \\
& =|q(t)-c|+\frac{\ell(t) \max \left\{\left|x\left(\alpha_{1}(t)\right)\right|,\left|x\left(\alpha_{2}(t)\right)\right|\right\}}{M+\max \left\{\left|x\left(\alpha_{1}(t)\right)\right|,\left|x\left(\alpha_{2}(t)\right)\right|\right\}}+v(t),
\end{aligned}
$$

for all $t \in \mathbb{R}_{+}$. This further implies that $\|Q X(t)-c\| \leq|q(t)-c|$ $+\ell(t)+v(t)$. Taking the limit superior in the above inequality, one obtains

$$
\begin{aligned}
\limsup _{t \rightarrow \infty}\|Q X(t)-c\| \leq & \limsup _{t \rightarrow \infty}|q(t)-c| \\
& +\limsup _{t \rightarrow \infty} \ell(t)+\limsup _{t \rightarrow \infty} v(t) .
\end{aligned}
$$

Further, using the measure of noncompactness $\mu_{c}$ defined by the formula (11) and keeping in mind the estimates (48) and (59), one obtains

$$
\begin{aligned}
\mu_{c}(Q X) & =\max \left\{\omega_{0}(Q X), \underset{t \rightarrow \infty}{\limsup }\|Q X(t)-c\|\right\} \\
& \leq \max \left\{\frac{L \omega_{0}(X)}{M+\omega_{0}(X)}, 0\right\} \leq \frac{L \max \left\{\omega_{0}(X), 0\right\}}{M+\max \left\{\omega_{0}(X), 0\right\}} \\
& \leq \frac{L \mu_{c}(X)}{M+\mu_{c}(X)}
\end{aligned}
$$

Since $L \leq M$ in view of assumption $\left(\mathrm{H}_{2}\right)$, from the above estimate one infers that $\mu_{c}(Q X) \leq \varphi\left(\mu_{c}(Q X)\right)$, where $\varphi(r)=$ $\mathrm{Lr} /(M+r)<r$ for $r>0$. Hence, apply Theorem 5 to deduce that the operator $Q$ has a fixed point $x$ in the ball $B_{r_{0}}$. Obviously $x$ is a solution of the fractional functional integral equation (21). Now, taking into account the description of sets belonging to ker $\mu_{c}$ (given in Section 2) one deduces that all solutions of (21) are uniformly globally asymptotically attractive on $\mathbb{R}_{+}$. This completes the proof.

Theorem 23. Under the hypotheses of Theorem 22 and $\left(H_{8}\right)$, (21) has at least one solution on $\mathbb{R}_{+}$. Moreover, solutions of (21) are uniformly globally asymptotically attractive and ultimately positive on $\mathbb{R}_{+}$.

Proof. The proof is similar to Theorem 21 with appropriate modifications. Now the desired conclusion follows an application of the measure of noncompactness $\mu_{c d}$ in $B C\left(\mathbb{R}_{+}, \mathbb{R}\right)$. This completes the proof.

\section{Applications}

In what follows, we show that the assumptions imposed in Theorems 19 and 21 admit some natural realizations. First, we indicate some possible forms for expressing the function $f_{1}$ that satisfies the hypothesis $\left(H_{2}\right)$. Define a class $\Theta$ of functions $\theta: \mathbb{R}_{+} \rightarrow \mathbb{R}_{+}$satisfying the following properties: (i) $\theta$ is continuous; (ii) $\theta$ is nondecreasing (iii) $\theta$ is subadditive, that is, $\theta(x+y) \leq \theta(x)+\theta(y)$ for all $x, y \in \mathbb{R}_{+}$.

Notice that if $\theta \in \Theta$, then after simple computation it can be shown that $|\theta(x)-\theta(y)| \leq \theta(|x-y|)$ for all $x, y \in \mathbb{R}_{+}$. Now consider the function $f_{1}: \mathbb{R}_{+} \times \mathbb{R} \times \mathbb{R} \rightarrow \mathbb{R}$ defined by

$$
f_{1}(t, x, y)=\ell(t) \frac{\theta_{1}(|x|)+\theta_{2}(|y|)}{2 M+\theta_{1}(|x|)+\theta_{2}(|y|)}+m(t),
$$

where the functions $\ell, m: \mathbb{R}_{+} \rightarrow \mathbb{R}$ are continuous and bounded on $\mathbb{R}_{+}$, that is, $\ell, m \in B C\left(\mathbb{R}_{+}, \mathbb{R}\right)$ with $\sup _{t \geq 0} \ell(t)=$ $L, \theta_{1}, \theta_{2} \in \Theta$ satisfying $\theta_{1}(r) \leq r, \theta_{2}(r) \leq r$, and $M$ is a positive constant such that $L \leq M$. It is shown as in the work of Dhage [11] that the function $f_{1}$ satisfies the condition (34) and consequently the hypothesis $\left(\mathrm{H}_{2}\right)$. There do exist functions $\theta$ given in the expression (61). Indeed, the following functions

$$
\begin{aligned}
& \theta(r)=r, \quad \theta(r)=\ln (1+r), \\
& \theta(r)=\arctan (r), \quad \theta(r)=2(\sqrt{1+r}-1)
\end{aligned}
$$

satisfy all the requirements of $\theta_{1}$ and $\theta_{2}$ given in (61) (cf. in the work of Banaś and Dhage [49]).

Finally, we provide two examples of the nonlinear fractional functional integral equations of the form (21) for which there are global attractive and ultimate positive solutions.

Example 24. Consider the following nonlinear functional integral equation:

$$
\begin{aligned}
x(t)= & \frac{1}{2} t e^{-t^{2} / 2} \\
& +\frac{t^{2}+1}{t^{2}+4} \times \frac{\arctan (|x(t)|)+\arctan (|x(2 t)|)}{9+\arctan (|x(t)|)+\arctan (|x(2 t)|)} \\
& +\frac{t+\sqrt{t}[x(t)+x(2 t)]}{\Gamma(2 / 3)} \\
& \times \int_{0}^{t} \frac{e^{-3 t-s \sqrt[3]{x^{2}(s)+x^{2}(2 s) / 4}}+1 /\left(10 t^{8 / 3}+1\right)}{(t-s)^{1 / 3}} \mathrm{~d} s .
\end{aligned}
$$

Observe that the above equation is a special case of the fractional functional integral equation (21). Indeed, if we put $\alpha=2 / 3$ and

$$
\begin{gathered}
\alpha_{1}(t)=\beta_{1}(t)=\gamma_{1}(t)=t, \\
\alpha_{2}(t)=\beta_{2}(t)=\gamma_{2}(t)=2 t, \quad q(t)=\frac{1}{2} t e^{-t^{2} / 2},
\end{gathered}
$$




$$
\begin{gathered}
f_{1}\left(t, x\left(\alpha_{1}(t)\right), x\left(\alpha_{2}(t)\right)\right) \\
=\frac{t^{2}+1}{t^{2}+4} \times \frac{\arctan (|x(t)|)+\arctan (|x(2 t)|)}{9+\arctan (|x(t)|)+\arctan (|x(2 t)|)}, \\
f_{2}\left(t, x\left(\beta_{1}(t)\right), x\left(\beta_{2}(t)\right)\right)=t+\sqrt{t}[x(t)+x(2 t)], \\
f_{3}\left(t, s, x\left(\gamma_{1}(s)\right), x\left(\gamma_{2}(s)\right)\right) \\
=e^{e^{-3 t-s} \sqrt[3]{x^{2}(s)+x^{2}(2 s) / 4}}+\frac{1}{10 t^{8 / 3}+1} .
\end{gathered}
$$

Obviously the functions $\alpha_{1}, \alpha_{2}, \beta_{1}, \beta_{2}$ and $\gamma_{1}, \gamma_{2}$ satisfy hypothesis $\left(H_{0}\right)$. Further notice that the function $q(t)=(1 / 2) t e^{-t^{2} / 2}$ is continuous and bounded on $\mathbb{R}_{+}$with $\|q\|=q(1)=(1 / 2) e^{-1 / 2}=0.30327 \ldots$. Thus assumption $\left(H_{1}\right)$ is satisfied. On the other hand, the function $f_{1}\left(t, x\left(\alpha_{1}(t)\right), x\left(\alpha_{2}(t)\right)\right)$ has the form (61) with $\ell(t)=\left(t^{2}+\right.$ $1) /\left(t^{2}+4\right)$. Moreover, $\theta(r)=\arctan (r)$ and $M=9 / 2$. Since $\|\ell\|=L=1$ one has that $L \leq M$. Additionally one has that the function $\theta$ satisfies above discussed requirements of the class of functions $\Theta$, so the function $f_{1}\left(t, x\left(\alpha_{1}(t)\right), x\left(\alpha_{2}(t)\right)\right)$ satisfies assumption $\left(\mathrm{H}_{2}\right)$ and $\left(\mathrm{H}_{4}\right)$.

Further observe that the function $f_{2}\left(t, x\left(\beta_{1}(t)\right), x\left(\beta_{2}(t)\right)\right)$ satisfies assumption $\left(H_{3}\right)$ with $m(t)=\sqrt{t}$ and $\left|F_{2}(t)\right|=$ $f_{2}(t, 0,0)=t$. Next, the function $f_{3}\left(t, s, x\left(\gamma_{1}(s)\right), x\left(\gamma_{2}(s)\right)\right)$ satisfies assumption $\left(H_{5}\right)$, where $n(t)=e^{-3 t}, \varphi(r)=\sqrt[3]{r^{2}} /$ 4 and $f_{3}(t, s, 0,0)=1 /\left(10 t^{8 / 3}+1\right)$. Thus $F_{3}(t)=1 /\left(10 t^{8 / 3}+1\right)$. To check that assumption $\left(H_{6}\right)$ is satisfied let us observe that the functions $a, b, c, d$ appearing in that assumption take the form

$$
\begin{array}{cr}
a(t)=2 t^{7 / 6} e^{-3 t}, & b(t)=\frac{2 t^{7 / 6}}{10 t^{8 / 3}+1}, \\
c(t)=t^{5 / 3} e^{-3 t}, & d(t)=\frac{t^{5 / 3}}{10 t^{8 / 3}+1} .
\end{array}
$$

Thus, it is easily seen that $a(t) \rightarrow 0$ as $t \rightarrow \infty$ and $A=$ $a(7 / 18)=2(7 / 18)^{7 / 6} e^{-7 / 6}=0.2069266 \ldots$ Further one has that $b(t) \rightarrow 0$ as $t \rightarrow \infty$ and $B=b\left((7 / 90)^{3 / 8}\right)=$ $0.0594821 \ldots$ It is also easy to check that $c(t) \rightarrow 0$ as $t \rightarrow$ $\infty$. Moreover, one has that $C=c(5 / 9)=(5 / 9)^{5 / 3} e^{-5 / 3}=$ $0.0709235 \ldots$. Also one sees that $d(t) \rightarrow 0$ as $t \rightarrow \infty$ and $D=d\left((1 / 6)^{3 / 8}\right)=0.1223733 \ldots$. Finally, let us note that the inequality from assumption $\left(H_{7}\right)$ has the form

$$
\frac{1}{2} e^{-1 / 2}+\frac{r}{9+r}+\frac{\operatorname{Ar} \varphi(2 r)+B r+C \varphi(2 r)+D}{\Gamma(5 / 3)}<r .
$$

Let us write this inequality in the form

$$
\begin{gathered}
\frac{1}{2} \Gamma\left(\frac{5}{3}\right) e^{-1 / 2}+\frac{\Gamma(5 / 3) r}{9+r}+\operatorname{Ar} \varphi(2 r) \\
+B r+C \varphi(2 r)+D<\Gamma\left(\frac{5}{3}\right) r .
\end{gathered}
$$

Denoting by $L(r)$ the left-hand side of this inequality, that is,

$$
\begin{aligned}
L(r)= & \frac{1}{2} \Gamma\left(\frac{5}{3}\right) e^{-1 / 2}+\frac{\Gamma(5 / 3) r}{9+r} \\
& +\operatorname{Ar} \varphi(2 r)+B r+C \varphi(2 r)+D
\end{aligned}
$$

and keeping in mind the above established values of $A, B, C$, $D$, for $r=1$ one obtains

$$
\begin{aligned}
L(1) & =\frac{1}{2} \Gamma\left(\frac{5}{3}\right) e^{-1 / 2}+\frac{\Gamma(5 / 3)}{10}+A \varphi(2)+B+C \varphi(2)+D \\
& =\frac{1}{2} \Gamma\left(\frac{5}{3}\right) 0.60653 \cdots+\frac{\Gamma(5 / 3)}{10}+0.963727 \ldots
\end{aligned}
$$

Hence, taking into account that $\Gamma(5 / 3)>0.8856$ (cf. [50]), one obtains that the number $r_{0}=1$ is a solution of the inequality (63).

Now, based on Theorem 19 one can conclude that the functional integral equation (63) has solutions in the space $B C\left(\mathbb{R}_{+}, \mathbb{R}\right)$ and all solutions of this equation are uniformly globally attractive on $\mathbb{R}_{+}$. Furthermore,

$$
\begin{aligned}
& \left|f_{3}\left(t, s, x\left(\gamma_{1}(s)\right), x\left(\gamma_{2}(s)\right)\right)\right| \\
& =e^{-3 t-s \sqrt[3]{x^{2}(s)+x^{2}(2 s)} / 4}+\frac{1}{10 t^{8 / 3}+1} \\
& \quad=f_{3}\left(t, s, x\left(\gamma_{1}(s)\right), x\left(\gamma_{2}(s)\right)\right)
\end{aligned}
$$

for all $t \in \mathbb{R}_{+}$and $x, y \in \mathbb{R}$. Hence the functions $q$ and $f_{3}\left(t, s, x\left(\gamma_{1}(s)\right), x\left(\gamma_{2}(s)\right)\right)$ satisfy the hypothesis $\left(H_{8}\right)$. Hence by Theorem 21, solutions of (63) are uniformly globally attractive and ultimately positive on $\mathbb{R}_{+}$.

Example 25. Consider the following nonlinear functional integral equation:

$$
\begin{aligned}
x(t)= & \frac{1}{2} t e^{-t^{2} / 2} \\
& +e^{-t} \frac{\arctan (|x(t)|)+\arctan (|x(2 t)|)}{9+\arctan (|x(t)|)+\arctan (|x(2 t)|)} \\
& +\frac{t+\sqrt{t}[x(t)+x(2 t)]}{\Gamma(2 / 3)} \\
& \times \int_{0}^{t} \frac{e^{-3 t-s \sqrt[3]{x^{2}(s)+x^{2}(2 s) / 4}}+1 /\left(10 t^{8 / 3}+1\right)}{(t-s)^{1 / 3}} \mathrm{~d} s .
\end{aligned}
$$

Observe that (71) is a special case of (21), where one has

$$
\begin{gathered}
\alpha_{1}(t)=\beta_{1}(t)=\gamma_{1}(t)=t, \\
\alpha_{2}(t)=\beta_{2}(t)=\gamma_{2}(t)=2 t, \quad q(t)=\frac{1}{2} t e^{-t^{2} / 2}, \\
f_{1}\left(t, x\left(\alpha_{1}(t)\right), x\left(\alpha_{2}(t)\right)\right) \\
=e^{-t} \frac{\arctan (|x(t)|)+\arctan (|x(2 t)|)}{9+\arctan (|x(t)|)+\arctan (|x(2 t)|)}, \\
f_{2}\left(t, x\left(\beta_{1}(t)\right), x\left(\beta_{2}(t)\right)\right)=t+\sqrt{t}[x(t)+x(2 t)], \\
f_{3}\left(t, s, x\left(\gamma_{1}(s)\right), x\left(\gamma_{2}(s)\right)\right) \\
=e^{-3 t-s \sqrt[3]{x^{2}(s)+x^{2}(2 s)} / 4}+\frac{1}{10 t^{8 / 3}+1} .
\end{gathered}
$$


Obviously, the functions $\alpha_{1}, \alpha_{2}, \beta_{1}, \beta_{2}$ and $\gamma_{1}, \gamma_{2}$ satisfy hypothesis $\left(H_{0}\right)$ and it is shown as in Example 24 that assumption $\left(H_{1}\right)$ is satisfied. Further, notice that the function $f_{1}\left(t, x\left(\alpha_{1}(t)\right), x\left(\alpha_{2}(t)\right)\right)$ has the form (61) with $\ell(t)=e^{-t}$ and $\lim _{t \rightarrow \infty} \ell(t)=\lim _{t \rightarrow \infty} e^{-t}=0$. Moreover, $\theta(r)=\arctan (r)$, $M=9 / 2$. Since $\|\ell\|=1$ one has that $L \leq M$. Additionally one has that $\theta \in \Theta$, so the function $f_{1}\left(t, x\left(\alpha_{1}(t)\right), x\left(\alpha_{2}(t)\right)\right)$ satisfies assumption $\left(H_{2}\right)$ and $\left(H_{4}\right)$.

Finally, it is shown as in Example 24 that the functions $f_{2}\left(t, x\left(\beta_{1}(t)\right), x\left(\beta_{2}(t)\right)\right)$ and $f_{3}\left(t, s, x\left(\gamma_{1}(s)\right), x\left(\gamma_{2}(s)\right)\right)$ are continuous on $\mathbb{R}_{+} \times \mathbb{R} \times \mathbb{R}$ and $\mathbb{R}_{+} \times \mathbb{R}_{+} \times \mathbb{R} \times \mathbb{R}$, respectively; moreover, they satisfy hypotheses $\left(\mathrm{H}_{3}\right)$ and $\left(\mathrm{H}_{5}\right)-\left(\mathrm{H}_{7}\right)$. Now, based on Theorem 22 one concludes that the fractional functional integral equation (61) has solutions in the space $B C\left(\mathbb{R}_{+}, \mathbb{R}\right)$ and all solutions of this equation are uniformly globally asymptotically attractive on $\mathbb{R}_{+}$. Furthermore,

$$
\begin{aligned}
& \left|f_{1}\left(t, x\left(\alpha_{1}(t)\right), x\left(\alpha_{2}(t)\right)\right)\right| \\
& \quad=e^{-t} \frac{\arctan (|x(t)|)+\arctan (|x(2 t)|)}{9+\arctan (|x(t)|)+\arctan (|x(2 t)|)} \\
& \quad=f_{1}\left(t, x\left(\alpha_{1}(t)\right), x\left(\alpha_{2}(t)\right)\right)
\end{aligned}
$$

for all $t \in \mathbb{R}_{+}$and $x, y \in \mathbb{R}$. Hence the functions $q$ and $f_{1}\left(t, x\left(\alpha_{1}(t)\right), x\left(\alpha_{2}(t)\right)\right)$ satisfy the hypotheses $\left(H_{8}\right)-\left(H_{11}\right)$. Hence by Theorem 23, solutions of (71) are uniformly globally asymptotically attractive and ultimately positive on $\mathbb{R}_{+}$.

Remark 26. Note that the global existence as well as attractivity and positivity results of (21) can be extended to the following fractional functional integral equation:

$$
\begin{gathered}
x(t)=q(t)+f_{1}\left(t, x\left(\alpha_{1}(t)\right), x\left(\alpha_{2}(t)\right), \ldots, x\left(\alpha_{n}(t)\right)\right) \\
+\frac{f_{2}\left(t, x\left(\beta_{1}(t)\right), x\left(\beta_{2}(t)\right), \ldots, x\left(\beta_{n}(t)\right)\right)}{\Gamma(\alpha)} \\
\times \int_{0}^{t}(t-s)^{\alpha-1} f_{3}\left(t, s, x\left(\gamma_{1}(s)\right), x\left(\gamma_{2}(s)\right), \ldots,\right. \\
\left.x\left(\gamma_{n}(s)\right)\right) \mathrm{d} s,
\end{gathered}
$$

with similar method under appropriate modifications. Then so obtained results are useful in determining the global attractivity and positivity and global asymptotic attractivity and positivity of solutions for the fractional functional integral equations defined, respectively, by

$$
\begin{aligned}
x(t)= & \frac{1}{2} t e^{-t^{2} / 2} \\
& +\frac{t^{2}+1}{t^{2}+4} \times \frac{\sum_{i=1}^{n} \arctan (|x(i t)|)}{9+\arctan \left(\sum_{i=1}^{n}|x(i t)|\right)} \\
& +\frac{t+\sqrt{t}\left[\sum_{i=1}^{n} x(i t)\right]}{\Gamma(2 / 3)} \\
& \times \int_{0}^{t} \frac{e^{-3 t-s \sqrt[3]{\sum_{i=1}^{n} x^{2}(i s) / 4}+1 /\left(10 t^{8 / 3}+1\right)}}{(t-s)^{1 / 3}} \mathrm{~d} s,
\end{aligned}
$$

$$
\begin{aligned}
x(t)= & \frac{1}{2} t e^{-t^{2} / 2} \\
& +e^{-t} \frac{\sum_{i=1}^{n} \arctan (|x(i t)|)}{9+\arctan \left(\sum_{i=1}^{n}(|x(i t)|)\right)}+\frac{t+\sqrt{t}\left[\sum_{i=1}^{n} x(i t)\right]}{\Gamma(2 / 3)} \\
& \times \int_{0}^{t} \frac{e^{-3 t-s \sqrt[3]{\sum_{i=1}^{n} x^{2}(i s) / 4}+1 /\left(10 t^{8 / 3}+1\right)}}{(t-s)^{1 / 3}} \mathrm{~d} s .
\end{aligned}
$$

\section{Acknowledgment}

This work is supported by the Guangdong Natural Science Foundation (no. S2011010004447).

\section{References}

[1] B. Ahmad and S. Sivasundaram, "Some existence results for fractional integro-differential equations with nonlinear conditions," Communications in Applied Analysis, vol. 12, no. 2, pp. 107-112, 2008.

[2] A. Anguraj, P. Karthikeyan, and G. M. N’Guérékata, "Nonlocal Cauchy problem for some fractional abstract integrodifferential equations in Banach spaces," Communications in Mathematical Analysis, vol. 6, no. 1, pp. 31-35, 2009.

[3] B. C. Dhage, "Asymptotic stability of nonlinear functional integral equations via measures of noncompactness," Communications on Applied Nonlinear Analysis, vol. 15, no. 2, pp. 89-101, 2008.

[4] K. Balachandran, S. Kiruthika, and J. J. Trujillo, "Existence results for fractional impulsive integrodifferential equations in Banach spaces," Communications in Nonlinear Science and Numerical Simulation, vol. 16, no. 4, pp. 1970-1977, 2011.

[5] J. Wang, Y. Zhou, and W. Wei, "A class of fractional delay nonlinear integrodifferential controlled systems in Banach spaces," Communications in Nonlinear Science and Numerical Simulation, vol. 16, no. 10, pp. 4049-4059, 2011.

[6] O. P. Agrawal, "Solution for a fractional diffusion-wave equation defined in a bounded domain," Nonlinear Dynamics, vol. 29, no. 1-4, pp. 145-155, 2002, Fractional order calculus and its applications.

[7] B. C. Dhage, "Local asymptotic attractivity for nonlinear quadratic functional integral equations," Nonlinear Analysis. Theory, Methods \& Applications A, vol. 70, no. 5, pp. 1912-1922, 2009.

[8] D. Băleanu and O. G. Mustafa, "On the global existence of solutions to a class of fractional differential equations," Computers \& Mathematics with Applications, vol. 59, no. 5, pp. 1835-1841, 2010.

[9] C. F. Li, X. N. Luo, and Y. Zhou, "Existence of positive solutions of the boundary value problem for nonlinear fractional differential equations," Computers \& Mathematics with Applications, vol. 59, no. 3, pp. 1363-1375, 2010.

[10] S. Zhang, "Positive solutions to singular boundary value problem for nonlinear fractional differential equation," Computers \& Mathematics with Applications, vol. 59, no. 3, pp. 1300-1309, 2010.

[11] B. C. Dhage, "Global attractivity results for nonlinear functional integral equations via a Krasnoselskii type fixed point theorem," 
Nonlinear Analysis. Theory, Methods \& Applications A, vol. 70, no. 7, pp. 2485-2493, 2009.

[12] M. Garg, A. Rao, and S. L. Kalla, "Fractional generalization of temperature field problem in oil strata," Matematichki Bilten, no. 30, pp. 71-84, 2006.

[13] Applications of Fractional Calculus in Physics, World Scientific, Singapore, 2000.

[14] S. G. Samko, A. A. Kilbas, and O. I. Marichev, Fractional Integrals and Derivatives, Gordon and Breach, Amsterdam, The Netherlands, 1993, Theory and Applications.

[15] S. Z. Rida, A. M. A. El-Sayed, and A. A. M. Arafa, "On the solutions of time-fractional reaction-diffusion equations," Communications in Nonlinear Science and Numerical Simulation, vol. 15, no. 12, pp. 3847-3854, 2010.

[16] D. Benson, The fractional advection-dispersion equation [Ph.D. thesis], University of Nevada, Reno, Nev, USA, 1998.

[17] R. Schumer, D. A. Benson, M. M. Meerschaert, and S. W. Wheatcraft, "Eulerian derivation of the fractional advectiondispersion equation," Journal of Contaminant Hydrology, vol. 48, no. 1-2, pp. 69-88, 2001.

[18] W. G. Glockle and T. F. Nonnenmacher, "A fractional calculus approach to self-similar protein dynamics," Biophysical Journal, vol. 68 , no. 1, pp. 46-53, 1995.

[19] R. Metzler, W. Schick, H. G. Kilian, and T. F. Nonnenmacher, "Relaxation in filled polymers: a fractional calculus approach," The Journal of Chemical Physics, vol. 103, no. 16, pp. 7180-7186, 1995.

[20] G. M. Mophou and G. M. N’Guérékata, "Existence of the mild solution for some fractional differential equations with nonlocal conditions," Semigroup Forum, vol. 79, no. 2, pp. 315-322, 2009.

[21] R. P. Agarwal, V. Lakshmikantham, and J. J. Nieto, "On the concept of solution for fractional differential equations with uncertainty," Nonlinear Analysis. Theory, Methods \& Applications A, vol. 72, no. 6, pp. 2859-2862, 2010.

[22] V. Lakshmikantham and A. S. Vatsala, "Basic theory of fractional differential equations," Nonlinear Analysis. Theory, Methods \& Applications A, vol. 69, no. 8, pp. 2677-2682, 2008.

[23] R. P. Agarwal, Y. Zhou, and Y. He, "Existence of fractional neutral functional differential equations," Computers \& Mathematics with Applications, vol. 59, no. 3, pp. 1095-1100, 2010.

[24] Y. Zhou, F. Jiao, and J. Li, "Existence and uniqueness for $p$-type fractional neutral differential equations," Nonlinear Analysis. Theory, Methods \& Applications A, vol. 71, no. 7-8, pp. 27242733, 2009.

[25] V. Lakshmikantham, "Theory of fractional functional differential equations," Nonlinear Analysis. Theory, Methods \& Applications A, vol. 69, no. 10, pp. 3337-3343, 2008.

[26] Y. Zhou and F. Jiao, "Existence of mild solutions for fractional neutral evolution equations," Computers \& Mathematics with Applications, vol. 59, no. 3, pp. 1063-1077, 2010.

[27] G. M. N’Guérékata, "A Cauchy problem for some fractional abstract differential equation with non local conditions," Nonlinear Analysis. Theory, Methods \& Applications A, vol. 70, no. 5, pp. 1873-1876, 2009.

[28] J. Cao, Q. Yang, and Z. Huang, "Optimal mild solutions and weighted pseudo-almost periodic classical solutions of fractional integro-differential equations," Nonlinear Analysis. Theory, Methods \& Applications A, vol. 74, no. 1, pp. 224-234, 2011.
[29] J. Cao, Q. Yang, and Z. Huang, "Existence of anti-periodic mild solutions for a class of semilinear fractional differential equations," Communications in Nonlinear Science and Numerical Simulation, vol. 17, no. 1, pp. 277-283, 2012.

[30] R.-N. Wang, D.-H. Chen, and T.-J. Xiao, "Abstract fractional Cauchy problems with almost sectorial operators," Journal of Differential Equations, vol. 252, no. 1, pp. 202-235, 2012.

[31] V. Obukhovskii and J.-C. Yao, "Some existence results for fractional functional differential equations," Fixed Point Theory, vol. 11, no. 1, pp. 85-96, 2010.

[32] S. D. Eidelman and A. N. Kochubei, "Cauchy problem for fractional diffusion equations," Journal of Differential Equations, vol. 199, no. 2, pp. 211-255, 2004.

[33] V. V. Anh and R. Mcvinish, "Fractional differential equations driven by Lévy noise," Journal of Applied Mathematics and Stochastic Analysis, vol. 16, no. 2, pp. 97-119, 2003.

[34] A. M. A. El-Sayed, "Nonlinear functional-differential equations of arbitrary orders," Nonlinear Analysis. Theory, Methods \& Applications A, vol. 33, no. 2, pp. 181-186, 1998.

[35] L. Yi and D. Shusen, "A class of analytic functions defined by fractional derivation," Journal of Mathematical Analysis and Applications, vol. 186, no. 2, pp. 504-513, 1994.

[36] V. Lakshmikantham and J. V. Devi, "Theory of fractional differential equations in a Banach space," European Journal of Pure and Applied Mathematics, vol. 1, no. 1, pp. 38-45, 2008.

[37] T. G. Bhaskar, V. Lakshmikantham, and S. Leela, "Fractional differential equations with a Krasnoselskii-Krein type condition," Nonlinear Analysis, vol. 3, no. 4, pp. 734-737, 2009.

[38] V. Lakshmikantham and S. Leela, "Nagumo-type uniqueness result for fractional differential equations," Nonlinear Analysis. Theory, Methods \& Applications A, vol. 71, no. 7-8, pp. 28862889, 2009.

[39] R. Akhmerov, M. Kamenskii, A. Potapov, A. Rodhina, and B. Sadovskii, Measures of Non-Compactness and Condensing Operators, Birkhauser, 1992.

[40] J. Appell, "Measures of noncompactness, condensing operators and fixed points: an application-oriented survey," Fixed Point Theory, vol. 6, no. 2, pp. 157-229, 2005.

[41] J. Banaś and K. Goebel, Measures of noncompactness in Banach spaces, vol. 60 of Lecture Notes in Pure and Applied Mathematics, Dekker, New York, NY, USA, 1980.

[42] M. Väth, Volterra and integral equations of vector functions, vol. 224, Marcel Dekker, New York, NY, USA, 2000.

[43] B. C. Dhage, "Attractivity and positivity results for nonlinear functional integral equations via measure of noncompactness," Differential Equations \& Applications, vol. 2, no. 3, pp. 299-318, 2010.

[44] B. C. Dhage, "Applicable fixed point theory in functional differential equations on unbounded intervals," Dynamic Systems and Applications, vol. 18, no. 3-4, pp. 701-724, 2009.

[45] A. A. Kilbas, H. M. Srivastava, and J. J. Trujillo, Theory and Applications of Fractional Differential Equations, vol. 204 of North-Holland Mathematics Studies, Elsevier, Amsterdam, The Netherlands, 2006.

[46] I. Podlubny, Fractional Differential Equations, vol. 198, Academic Press, San Diego, Calif, USA, 1999.

[47] J. Banaś and D. O’Regan, “On existence and local attractivity of solutions of a quadratic Volterra integral equation of fractional order," Journal of Mathematical Analysis and Applications, vol. 345, no. 1, pp. 573-582, 2008. 
[48] K. Balachandran, J. Y. Park, and M. D. Julie, "On local attractivity of solutions of a functional integral equation of fractional order with deviating arguments," Communications in Nonlinear Science and Numerical Simulation, vol. 15, no. 10, pp. 2809-2817, 2010.

[49] J. Banaś and B. C. Dhage, "Global asymptotic stability of solutions of a functional integral equation," Nonlinear Analysis. Theory, Methods \& Applications A, vol. 69, no. 7, pp. 1945-1952, 2008.

[50] G. Fichtenholz, Differential and Integral Calculus, II, PWN, Warsaw, Poland, 1980. 


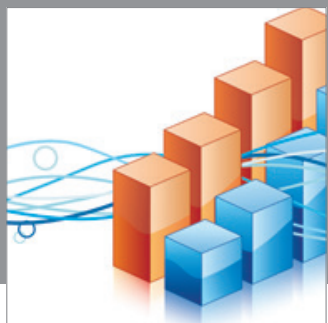

Advances in

Operations Research

mansans

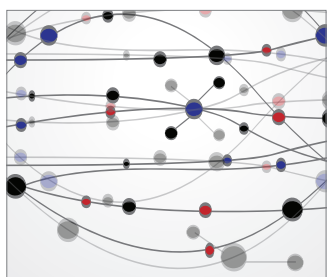

The Scientific World Journal
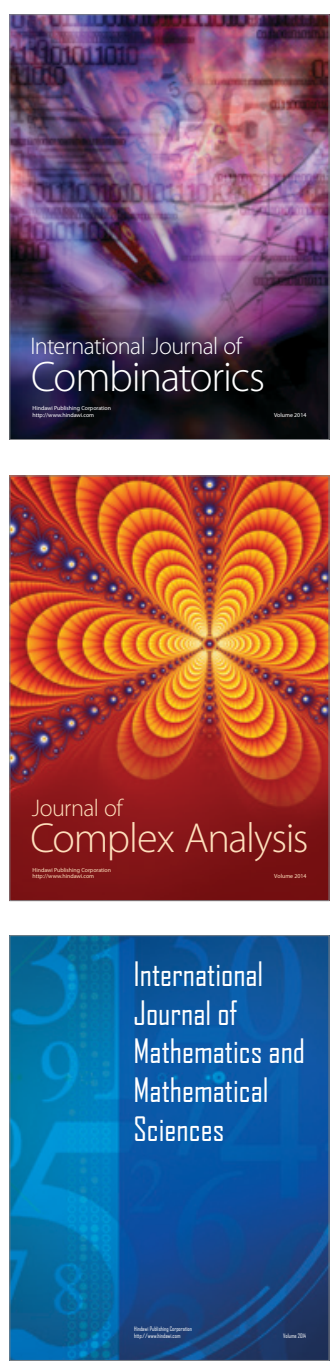
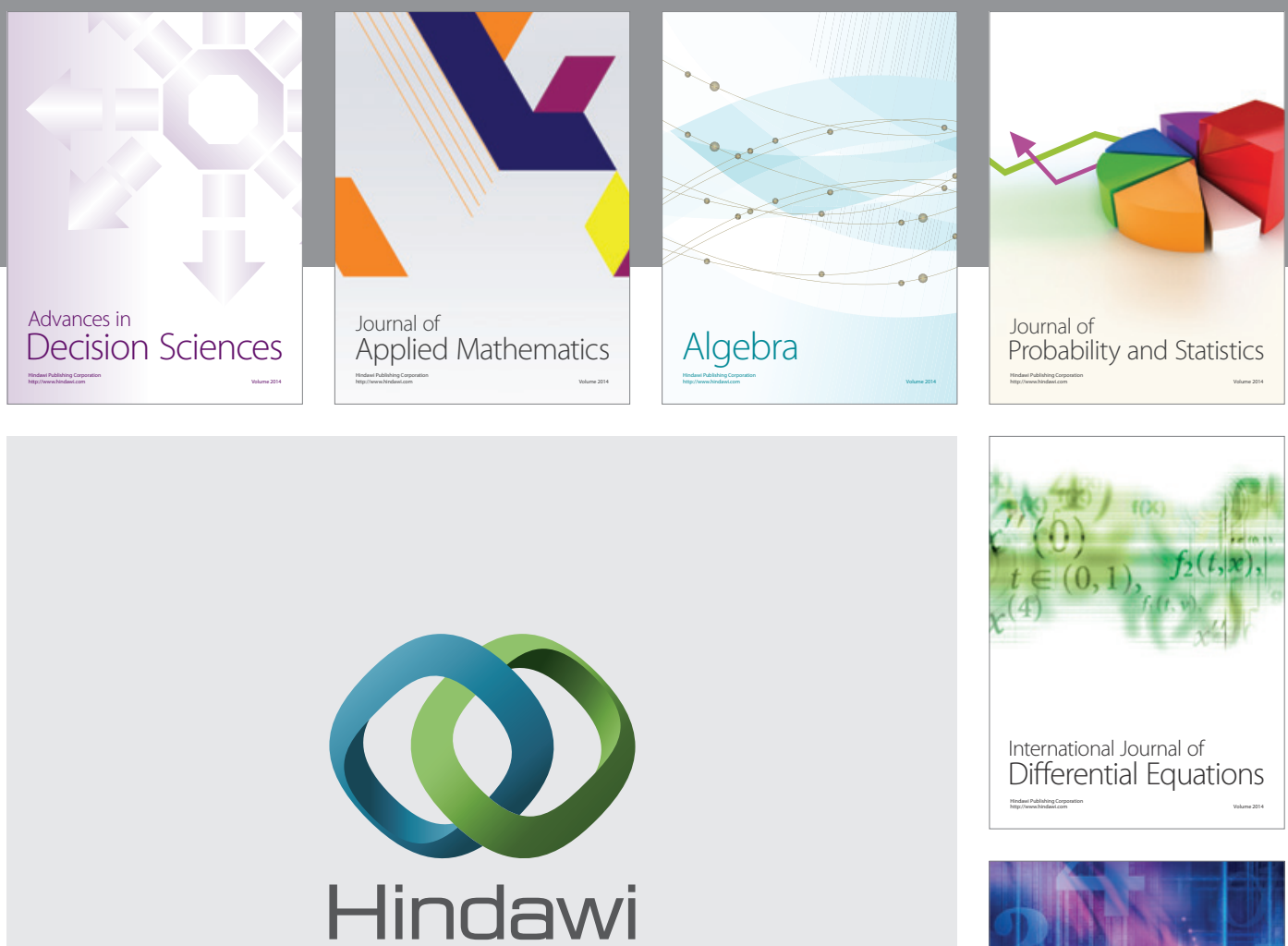

Submit your manuscripts at http://www.hindawi.com
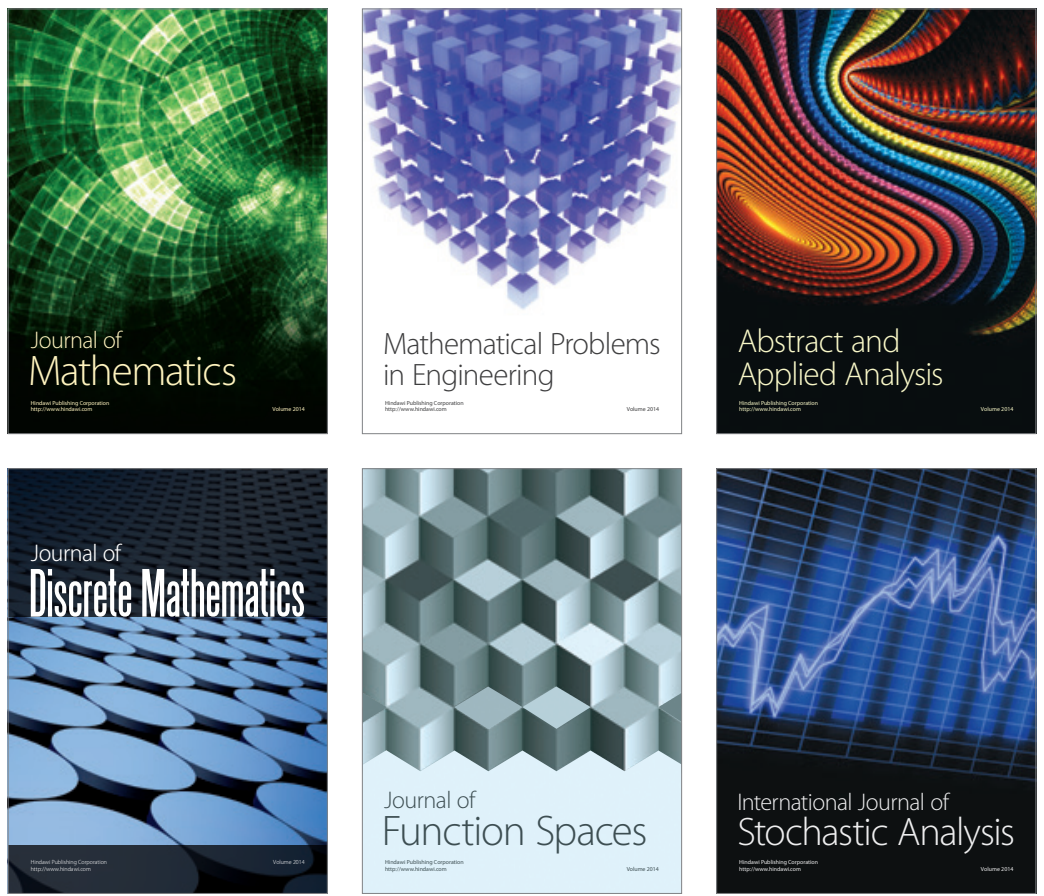

Journal of

Function Spaces

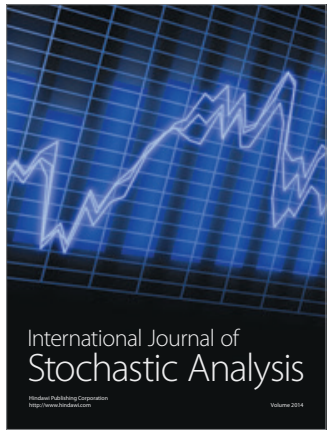

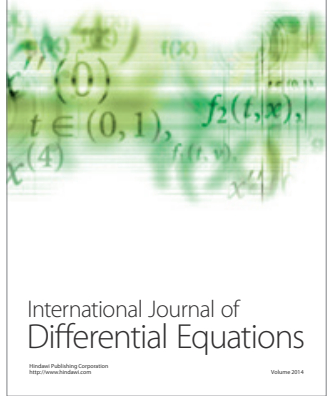
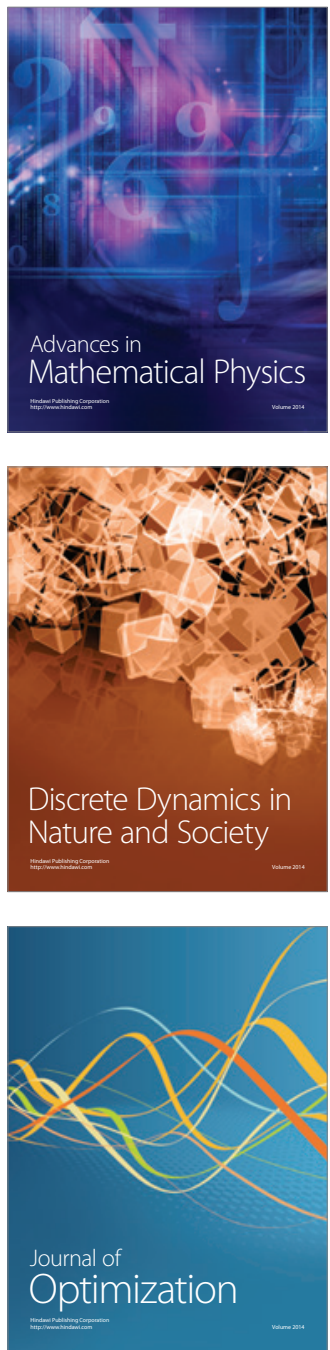